Supporting Information

\title{
Supramolecular Gold Stripping from Activated Carbon Using $\alpha$-Cyclodextrin
}

Wenqi Liu, ${ }^{1}$ Leighton O. Jones, ${ }^{1}$ Huang Wu, ${ }^{1}$ Charlotte L. Stern, ${ }^{1}$ Rebecca Sponenburg, ${ }^{2}$ George C. Schatz ${ }^{1}$ and J. Fraser Stoddart ${ }^{1,3 *}$

1. Department of Chemistry, 2145 Sheridan Road, Northwestern University, Evanston, IL 60208, USA

2. Quantitative Bio-Element Imaging Center, Northwestern University, Evanston, IL, 60208, USA

3. School of Chemistry, University of New South Wales, Sydney, NSW 2052, Australia

Email: stoddart@northwestern.edu

\section{Table of Contents}

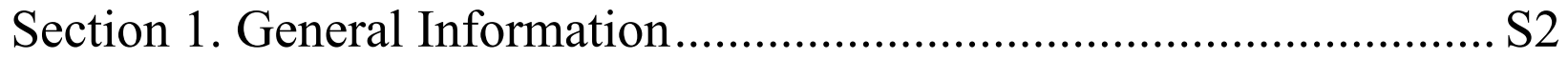

Section 2. Crystallographic Analyses .................................................. S3

Section 3. Computational Investigations .......................................... S11

Section 4. Binding Studies Using NMR Spectroscopy....................... S28

Section 5. Binding Studies Using Isothermal Titration Calorimetry ... S34

Section 6. Gold and Silver Stripping ………….............................. S37

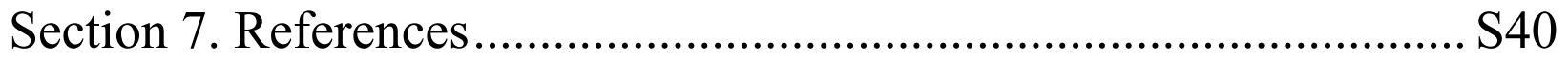




\section{Section 1. General Information}

Commercially available solvents and chemicals were purchased from Sigma-Aldrich and Fisher Scientific and used without further purification unless otherwise stated. Nuclear magnetic resonance (NMR) spectra were recorded on a Bruker AVANCE III $600 \mathrm{MHz}$ spectrometer. Chemical shifts are reported in ppm relative to the signals corresponding to the residual nondeuterated solvents. Single crystal data were obtained on a XtaLAB Synergy, Single source at offset/far, HyPix diffractometer. ICP-MS was performed on a computer-controlled (QTEGRA software) Thermo iCapQ ICP-MS (Thermo Fisher Scientific, Waltham, MA, USA) operating in STD mode and equipped with an ESI SC-2DX PrepFAST autosampler (Omaha, NE, USA). Detailed experimental procedures are provided in the appropriate sections that follow this one. Activated carbon (Part number: 05105, Lot code: 1136095) were purchased from Fluka with the following specifications:

\begin{tabular}{|l|c|l|l|}
\hline Loss on drying $\left(110{ }^{\circ} \mathrm{C}\right)$ & $<15 \%$ & $\mathrm{Co}$ & $<0.001 \%$ \\
\hline Ash $\left(600{ }^{\circ} \mathrm{C}\right)$ & $<0.1 \%$ & $\mathrm{Cr}$ & $<0.001 \%$ \\
\hline $\begin{array}{l}\text { Iodine-adsorption }(0.05 \\
\left.\text { mol } \mathrm{I}_{2} / \mathrm{I}\right)\end{array}$ & $>70 \mathrm{ml} / \mathrm{g}$ & $\mathrm{Cu}$ & $<0.001 \%$ \\
\hline $\begin{array}{l}\text { Methylene blue- } \\
\text { adsorption }(0.15 \% \text { sol. })\end{array}$ & $>12 \mathrm{ml} / 0.1 \mathrm{~g}$ & $\mathrm{Fe}$ & $<0.03 \%$ \\
\hline $\begin{array}{l}\text { Soluble material in } \\
\text { ethanol }\end{array}$ & $<0.2 \%$ & $\mathrm{~K}$ & $<0.02 \%$ \\
\hline Soluble material in $\mathrm{HCl}$ & $<1 \%$ & $\mathrm{Mg}$ & $<0.02 \%$ \\
\hline Water-soluble matter & $<0.5 \%$ & $\mathrm{Mn}$ & $<0.005 \%$ \\
\hline Chloride $(\mathrm{Cl})$ & $<0.5 \%$ & $\mathrm{Na}$ & $<0.05 \%$ \\
\hline Sulfate $\left(\mathrm{SO}_{4}\right)$ & $<0.5 \%$ & $\mathrm{Ni}$ & $<0.001 \%$ \\
\hline $\mathrm{Ca}$ & $<0.1 \%$ & $\mathrm{~Pb}$ & $<0.001 \%$ \\
\hline $\mathrm{Cd}$ & $<0.001 \%$ & $\mathrm{Zn}$ & $<0.001 \%$ \\
\hline
\end{tabular}




\section{Section 2. Crystallographic Analyses}

\section{(1) Crystal superstructure of $\mathrm{KAu}(\mathbf{C N})_{2} \subset \boldsymbol{\alpha}-\mathbf{C D}(\mathrm{CCDC}: 2040417)$}

(a) Method: Colorless block-like single crystals were obtained by slow evaporation of an aqueous solution $(300 \mu \mathrm{L})$ containing $\mathrm{KAu}(\mathrm{CN})_{2}(8.9 \mathrm{mg})$ and $\alpha-\mathrm{CD}(30 \mathrm{mg})$ at room temperature for 30 days. A suitable crystal was selected, and the crystal was mounted on a MITIGEN holder with Paratone oil on a XtaLAB Synergy, Single source at home/near, HyPix diffractometer. The crystal was kept at $100.02(10) \mathrm{K}$ during data collection. Using Olex2 ${ }^{\mathrm{S} 1}$, the superstructure was solved with the ShelXT ${ }^{\mathrm{S} 2}$ structure solution program using Intrinsic Phasing and refined with the $\mathrm{XL}^{\mathrm{S} 3}$ refinement package using Least Squares minimization.

(b) Crystal Parameters: Empirical formula $=\mathrm{C}_{38} \mathrm{H}_{74} \mathrm{AuKN}_{2} \mathrm{O}_{37}$, formula weight $=1387.05$, orthorhombic, space group $P 2{ }_{1} 2{ }_{1}{ }_{1}$ (no. 19), $a=13.99063(13), b=23.4405(2), c=51.2967(4) \AA$, $V=16822.6(3) \AA^{3}, Z=12, T=100.02(10) \mathrm{K}, \mu(\mathrm{CuK} \alpha)=6.517 \mathrm{~mm}^{-1}, D_{\text {calc }}=1.643 \mathrm{~g} / \mathrm{mm}^{3}, 97178$ reflections measured $(6.548 \leq 2 \Theta \leq 160.584), 34431$ unique $\left(R_{\text {int }}=0.0607, R_{\text {sigma }}=0.0620\right)$ which were used in all calculations. The final $R_{1}$ was $0.0588(I>2 \sigma(I))$ and $w R_{2}$ was 0.1474 (all data).

(c) Refinement Details: The enhanced rigid-bond restraint (SHELX keyword RIGU) was applied ${ }^{\mathrm{S} 4}$ globally.

(d) Solvent Treatment Details: The solvent masking procedure implemented in Olex2 was used to remove the electronic contribution of solvent molecules from the refinement. As the exact solvent content is unknown, only the atoms used in the refinement model are reported in the formula here. Total solvent-accessible volume $/$ cell $=889.1 \AA^{3}[5.3 \%]$ Total electron count $/$ cell = 175.9 
(a)
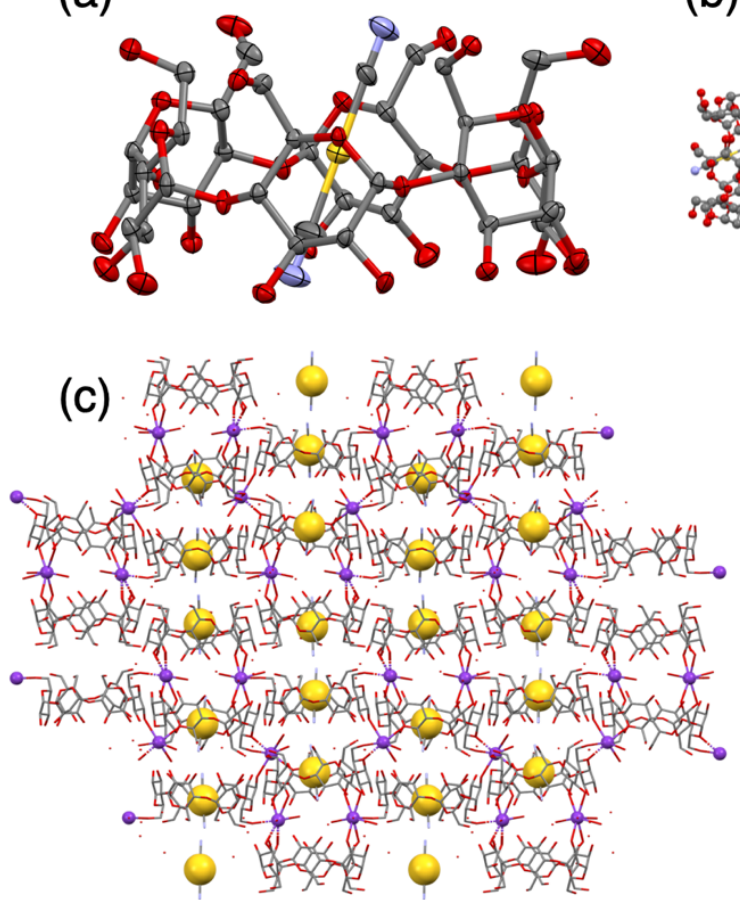

(b)

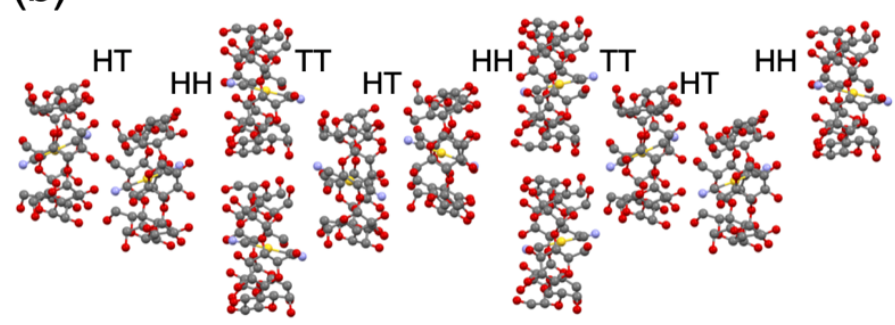

(d)

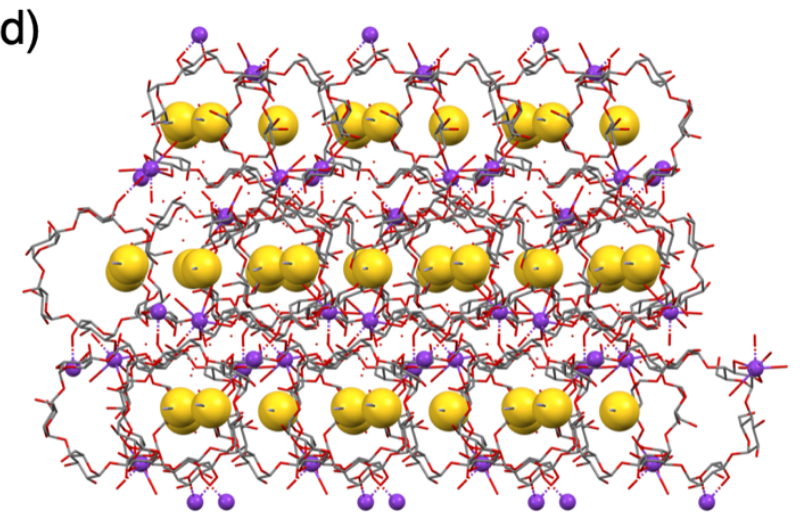

Figure S1. (a) Thermal ellipsoids representation of the solid-state superstructures of $\mathrm{KAu}(\mathrm{CN})_{2}$ $\subset(\alpha-C D)$ obtained from single-crystal X-ray crystallography. The thermal ellipsoids are shown at a $50 \%$ probability level. (b) Ball-and-stick representation of the packing of the $\alpha$-CDs showing the order of HT / HH / TT where H (head) represents the secondary face and T (tail) represents the primary face of $\alpha$-CD. Side-on view (c) and top view (d) of crystal packing highlighting the position of potassium (purple sphere) and gold (yellow sphere) ions. Hydrogens and solvents are omitted for the sake of clarity
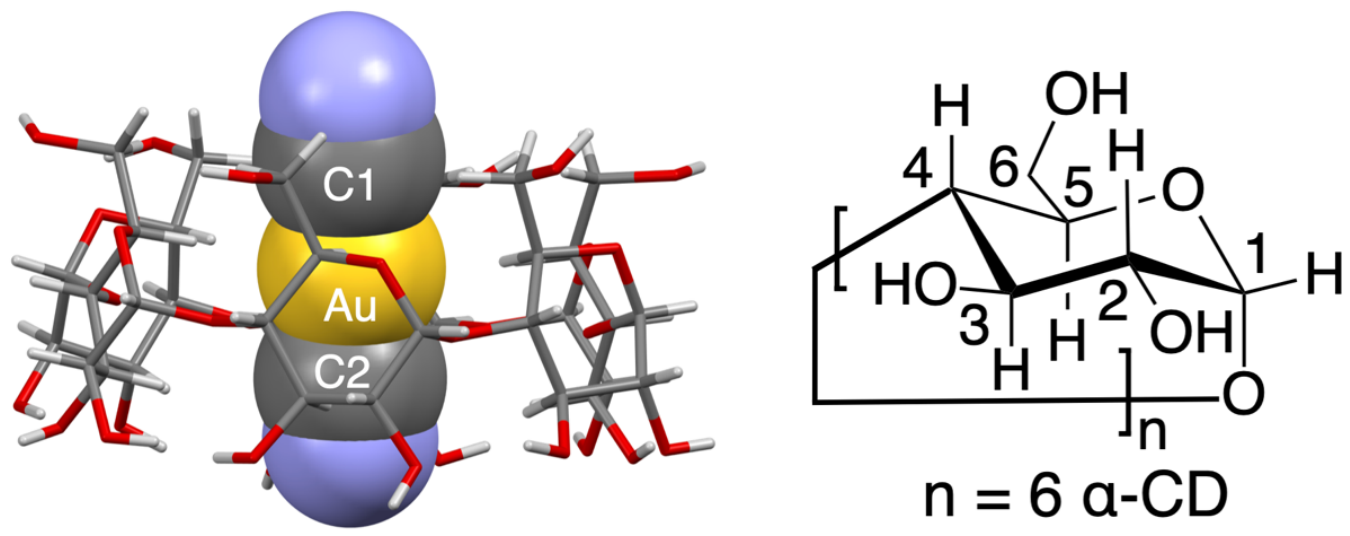

Figure S2. Labeling for $\mathrm{Au}(\mathrm{CN})_{2}{ }^{-}$and $\alpha-\mathrm{CD}$ in the solid-state superstructure of $\mathrm{KAu}(\mathrm{CN})_{2} \subset \alpha-$ CD 
Table S1. Distances between $\mathrm{Au}(\mathrm{CN})_{2}{ }^{-}$and the inner protons $\mathrm{H}-3, \mathrm{H}-5$ and $\mathrm{H}-6$ in each of the six glucose subunits of $\alpha-C D$. Labels are shown in Figure S2

\begin{tabular}{ccccccc}
\hline $\begin{array}{c}\text { Distances } \\
\AA\end{array}$ & Unit 1 & Unit 2 & Unit 3 & Unit 4 & Unit5 & Unit 6 \\
\hline $\mathrm{C} 1-\mathrm{H} 6$ & $3.7 / 3.8$ & $3.4 / 3.8$ & $3.9 / 4.6$ & $5.0 / 5.5$ & $4.8 / 4.6$ & $3.8 / 3.8$ \\
$\mathrm{Au}-\mathrm{H} 5$ & 3.3 & 3.2 & 3.3 & 3.6 & 3.3 & 3.1 \\
$\mathrm{C} 2-\mathrm{H} 3$ & 4.0 & 4.3 & 3.4 & 3.1 & 3.8 & 3.8 \\
\hline
\end{tabular}

(2) Crystal superstructure of $\mathrm{KAu}(\mathbf{C N})_{2} \subset 2 \boldsymbol{\alpha}-\mathbf{C D}(\mathrm{CCDC}: 2040418)$

(a) Method: Colorless block-like single crystals were obtained by slow diffusion of EtOH into an aqueous solution $(300 \mu \mathrm{L})$ containing $\mathrm{KAu}(\mathrm{CN})_{2}(8.9 \mathrm{mg})$ and $\alpha-\mathrm{CD}(30 \mathrm{mg})$ at room temperature for 14 days. A suitable crystal was selected, and the crystal was mounted on a MITIGEN holder with Paratone oil on a XtaLAB Synergy, Single source at offset/far, HyPix diffractometer. The crystal was kept at 100.0(2) K during data collection. Using Olex $2^{\mathrm{S} 1}$, the superstructure was solved with the $\mathrm{XT}^{\mathrm{S} 2}$ structure solution program using Intrinsic Phasing and refined with the XL refinement package ${ }^{\mathrm{S} 3}$ using Least Squares minimization.

(b) Crystal Parameters: Empirical formula $=\mathrm{C}_{39} \mathrm{H}_{74} \mathrm{Au}_{0.5} \mathrm{~K}_{0.5} \mathrm{NO}_{35}$, formula weight $=1235.02$, triclinic, space group $P \overline{1}$ (no. 1), $a=13.7130(5), b=13.7765(4), c=15.6190(4) \AA, \alpha=86.125(2)$, $\beta=86.183(3), \gamma=60.219(3)^{\circ}, V=2553.29(15) \AA^{3}, Z=2, T=100.0(2) \mathrm{K}, \mu(\mathrm{MoK} \alpha)=1.600 \mathrm{~mm}^{-1}$, $D_{\text {calc }}=1.606 \mathrm{~g} / \mathrm{mm}^{3}, 54235$ reflections measured $(4.214 \leq 2 \Theta \leq 66.388), 25086$ unique $\left(R_{\text {int }}=\right.$ $\left.0.0503, R_{\text {sigma }}=0.0804\right)$ which were used in all calculations. The final $R_{1}$ was $0.0571(I>2 \sigma(I))$ and $w R_{2}$ was 0.1383 (all data).

(c) Refinement Details: The enhanced rigid-bond restraint (SHELX keyword RIGU) was applied ${ }^{\mathrm{S} 4}$ on the disordered gold cyanide. The gold was partially disordered (about 3\% in each site). The gold atoms were refined with a group anisotropic displacement parameter. Three reflections were omitted for being behind the beam stop.

(d) Solvent Treatment Details: N/A 
(a)

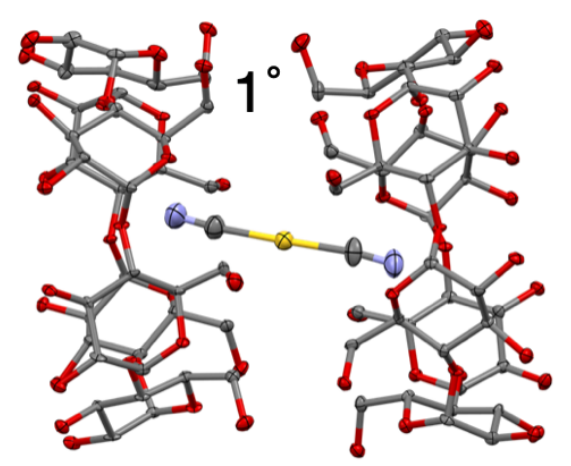

(c)

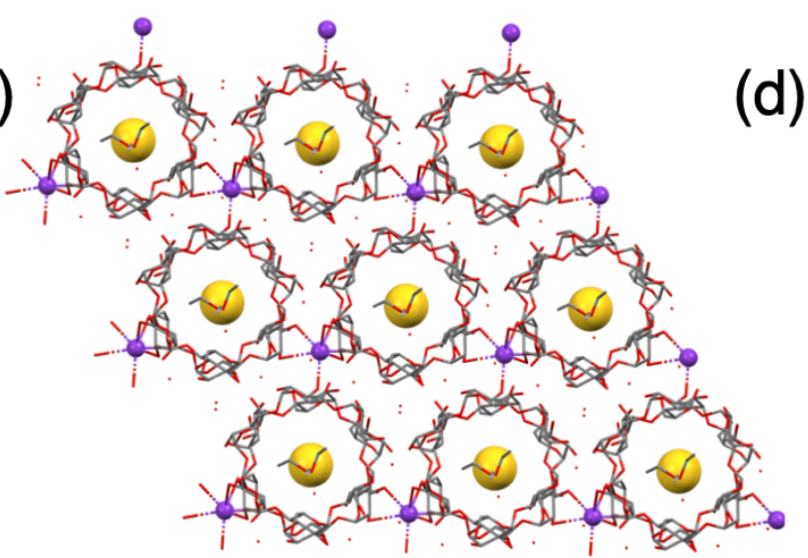

(b)
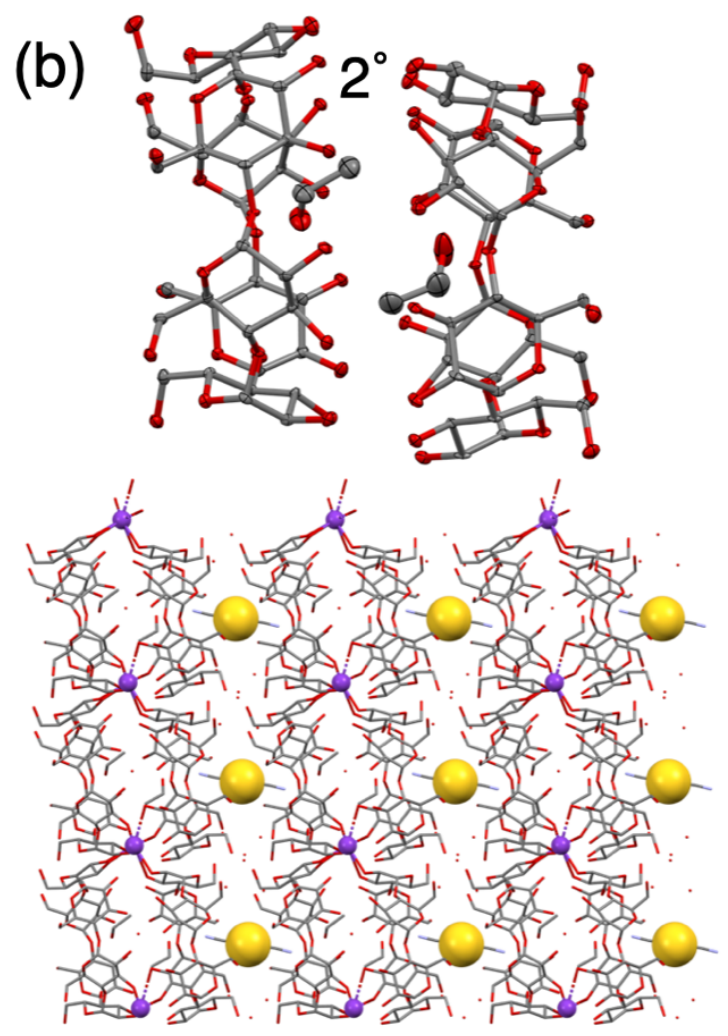

(e)

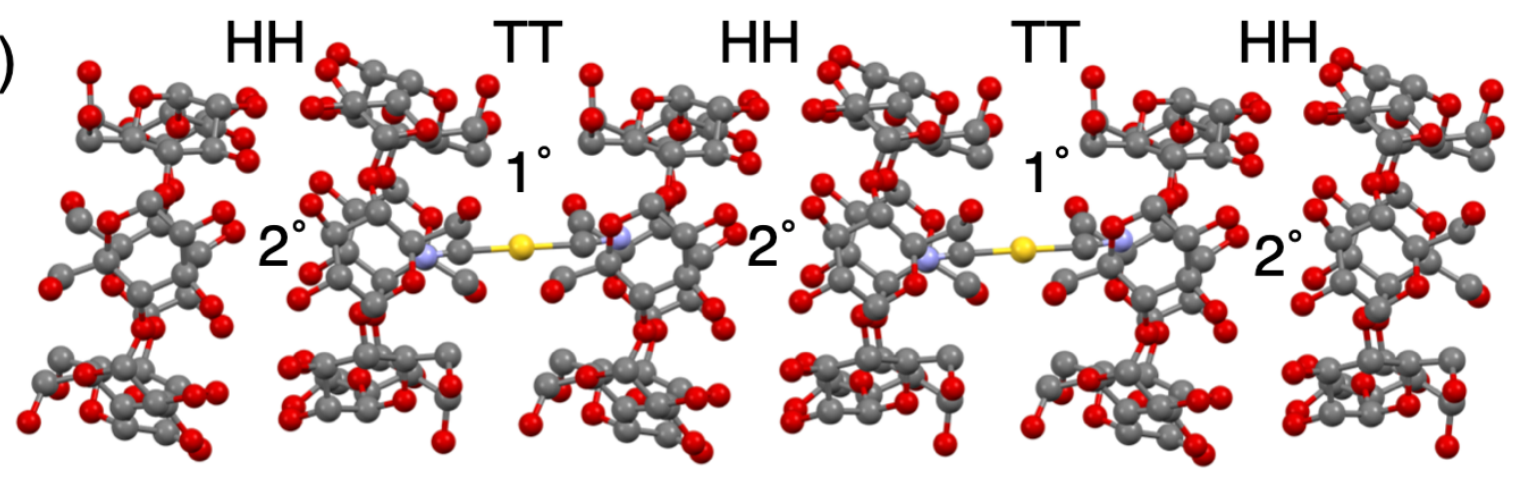

Figure S3. Thermal ellipsoids representation of the solid-state superstructures of $\operatorname{KAu}(\mathrm{CN})_{2} \subset(\alpha$ $\mathrm{CD})_{2}$ obtained from single-crystal X-ray crystallography, showing that the $\mathrm{KAu}(\mathrm{CN})_{2}$ is located (a) between the two primary faces while two EtOH molecules are revealed (b) at the two secondary faces of $\alpha$-CD. Thermal ellipsoids are shown at a 50\% probability level. Top view (c) and side-on view (d) of crystal packing highlighting the position of potassium (purple sphere) and gold (yellow sphere) ions. (e) The packing of the $\alpha$-CDs showing the order of HH / TT / HH / TT where H (head) represents the secondary face and T (tail) represents the primary face of $\alpha$-CD. Hydrogens and solvents are omitted for the sake of clarity 

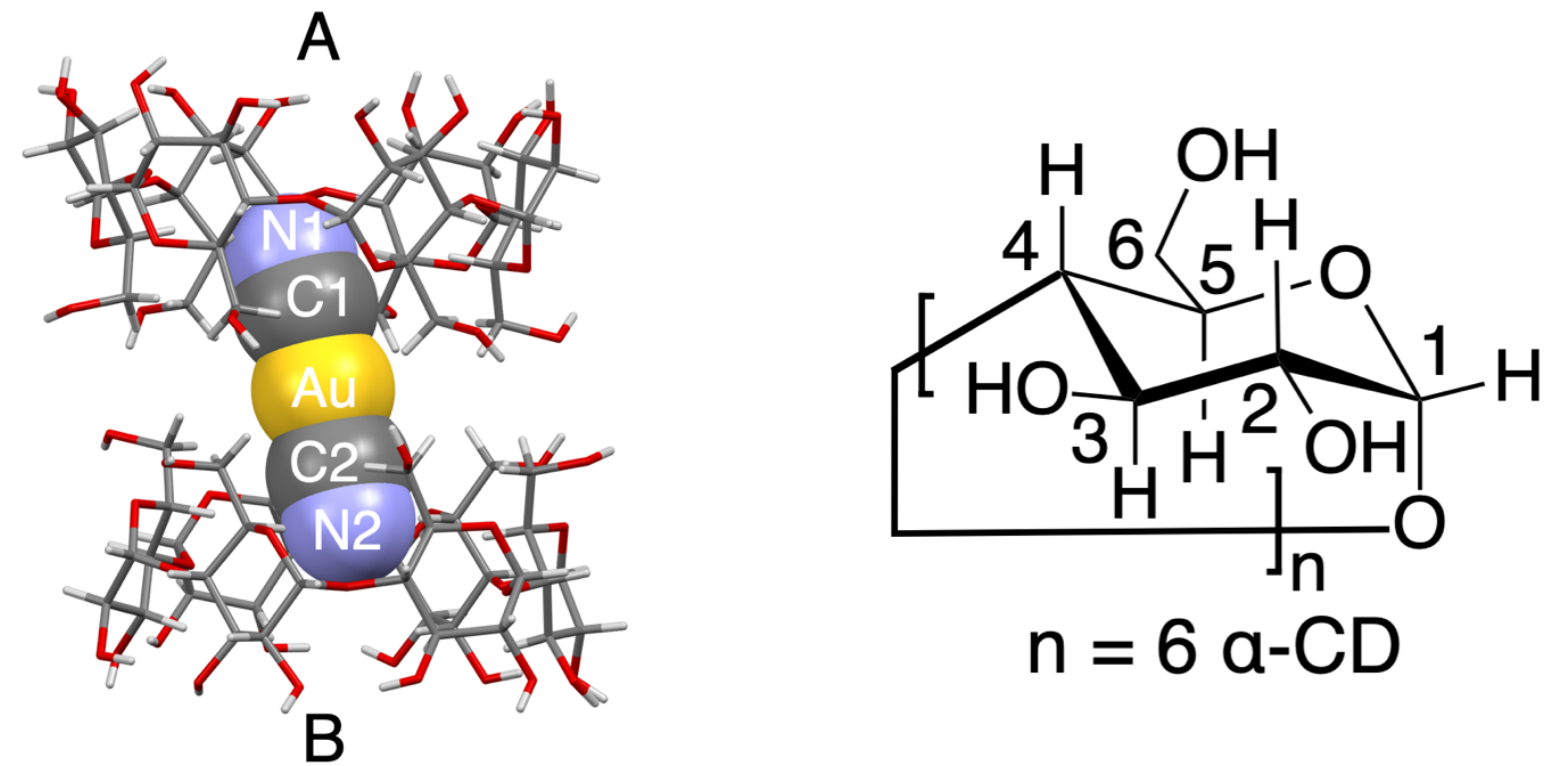

Figure S4. Labeling for $\mathrm{Au}(\mathrm{CN})_{2}^{-}$and $\alpha$-cyclodextrin in the solid-state superstructure of $\mathrm{KAu}(\mathrm{CN})_{2} \subset(\alpha-\mathrm{CD})_{2}$. The top $\alpha-\mathrm{CD}$ is labeled as $\mathrm{A}$ and the bottom $\alpha-\mathrm{CD}$ is labeled as $\mathrm{B}$

Table S2. Distances between $\mathrm{Au}(\mathrm{CN})_{2}{ }^{-}$and the inner protons $\mathrm{H}-5$ and $\mathrm{H}-6$ in each of the six glucose subunits of the two $\alpha$-CDs (A and B). Labels are shown in Figure S4

\begin{tabular}{ccccccc}
\hline Distances $\AA$ & Unit 1 & Unit 2 & Unit 3 & Unit 4 & Unit5 & Unit 6 \\
\hline C1-AH6 & $4.0 / 3.5$ & $4.1 / 4.1$ & $4.2 / 4.4$ & $4.7 / 4.5$ & $4.0 / 3.9$ & $3.9 / 3.7$ \\
Au-AH6 & $4.0 / 3.7$ & $3.5 / 3.9$ & $3.8 / 4.4$ & $4.8 / 5.0$ & $4.7 / 4.9$ & $4.5 / 4.5$ \\
N1-AH5 & 3.2 & 3.6 & 3.7 & 3.5 & 2.8 & 2.8 \\
C2-BH6 & $3.6 / 4.2$ & $3.6 / 4.1$ & $4.0 / 4.3$ & $4.7 / 4.9$ & $4.6 / 4.2$ & $4.0 / 3.9$ \\
Au-BH6 & $4.3 / 3.8$ & $4.4 / 4.7$ & $4.9 / 4.8$ & $5.1 / 4.9$ & $4.5 / 3.7$ & $3.7 / 3.4$ \\
N2-BH5 & 2.8 & 2.8 & 3.2 & 3.9 & 3.8 & 3.2 \\
\hline
\end{tabular}




\section{(3) Crystal superstructure of $\mathrm{KAg}(\mathbf{C N})_{2} \subset 2 \boldsymbol{\alpha}-\mathbf{C D}(\mathrm{CCDC}: 2040419)$}

(a) Method: Colorless block-like single crystals were obtained by slow diffusion of EtOH into an aqueous solution $(300 \mu \mathrm{L})$ containing $\mathrm{KAg}(\mathrm{CN})_{2}(6.2 \mathrm{mg})$ and $\alpha-\mathrm{CD}(30 \mathrm{mg})$ at room temperature during 14 days. A suitable crystal was selected, and the crystal was mounted on a MITIGEN holder with Paratone oil on a XtaLAB Synergy, Single source at offset/far, HyPix diffractometer. The crystal was kept at $99.98(17) \mathrm{K}$ during data collection. Using Olex $2^{\mathrm{S} 1}$, the superstructure was solved with the $\mathrm{XT}^{\mathrm{S} 2}$ structure solution program using Intrinsic Phasing and refined with the XL refinement package ${ }^{\mathrm{S} 3}$ using Least Squares minimization.

(b) Crystal Parameters: Empirical formula $=\mathrm{C}_{78} \mathrm{H}_{148} \mathrm{AgKN}_{2} \mathrm{O}_{70}$, formula weight $=2380.95$, triclinic, space group $P \overline{1}$ (no. 1), $a=13.7332(5), b=13.7848(5), c=15.6408(7) \AA, \alpha=86.135(4)$, $\beta=86.215(3), \gamma=60.269(4)^{\circ}, V=2563.54(19) \AA^{3}, Z=1, T=99.98(17) \mathrm{K}, \mu(\mathrm{MoK} \alpha)=0.356 \mathrm{~mm}^{-1}$, $D_{\text {calc }}=1.542 \mathrm{~g} / \mathrm{mm}^{3}, 17511$ reflections measured $(4.15 \leq 2 \Theta \leq 59.436), 12082$ unique $\left(R_{\text {int }}=\right.$ $\left.0.0362, R_{\text {sigma }}=0.0527\right)$ which were used in all calculations. The final $R_{1}$ was $0.0755(I>2 \sigma(I))$ and $w R_{2}$ was 0.1988 (all data).

(c) Refinement Details: The enhanced rigid-bond restraint (SHELX keyword RIGU) was applied ${ }^{\mathrm{S} 4}$ on the disordered silver cyanide. The silver atoms were partially disordered (about $4 \%$ in each site). The silver atoms were refined with a group anisotropic displacement parameter. C31 was refined so that its displacement parameter was approximate isotropic.

(d) Solvent Treatment Details: N/A 
(a)

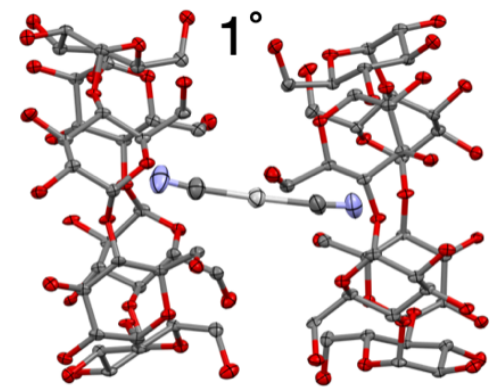

(c)

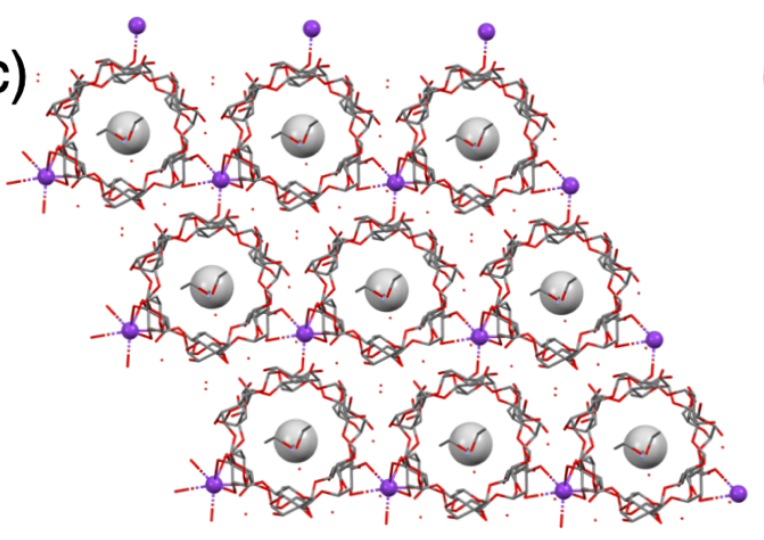

(b)

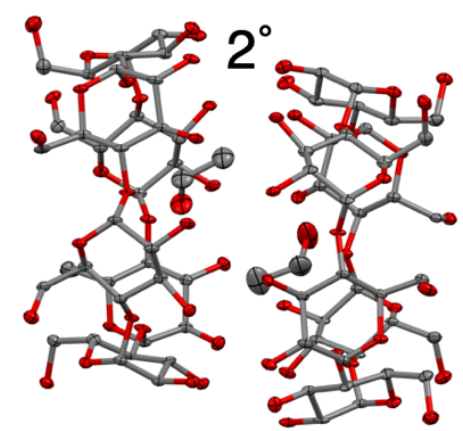

(d)

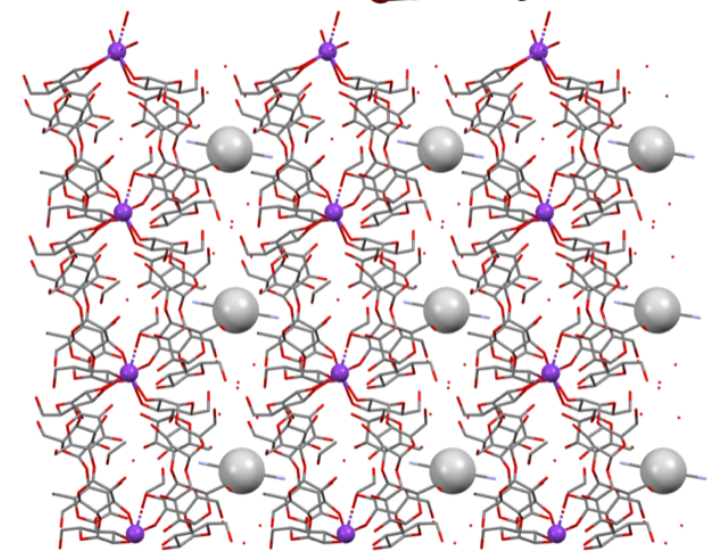

(e)

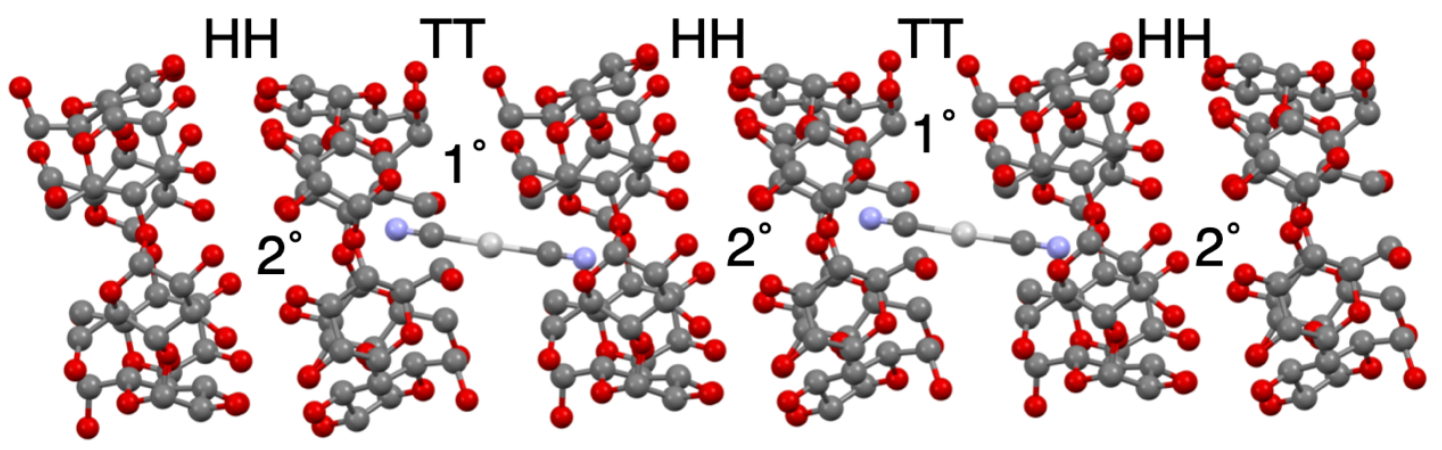

Figure S5. Thermal ellipsoids representation of the solid-state superstructures of $\operatorname{KAg}(\mathrm{CN})_{2} \subset(\alpha$ -

$\mathrm{CD})_{2}$ obtained from single-crystal X-ray crystallography showing that the $\mathrm{KAg}(\mathrm{CN})_{2}$ is located (a) between the two primary faces while two EtOH molecules are revealed (b) at the two secondary faces of $\alpha-\mathrm{CD}$. Thermal ellipsoids are shown at a 50\% probability level. Top view (c) and side-on view (d) of crystal packing highlighting the position of potassium (purple sphere) and silver (silver sphere) ions. (e) The packing of the $\alpha$-CDs showing the order of HH / TT / HH / TT where H (head) represents the secondary face and $\mathrm{T}$ (tail) represents the primary face of $\alpha$-CD. Hydrogens and solvents are omitted for the sake of clarity 

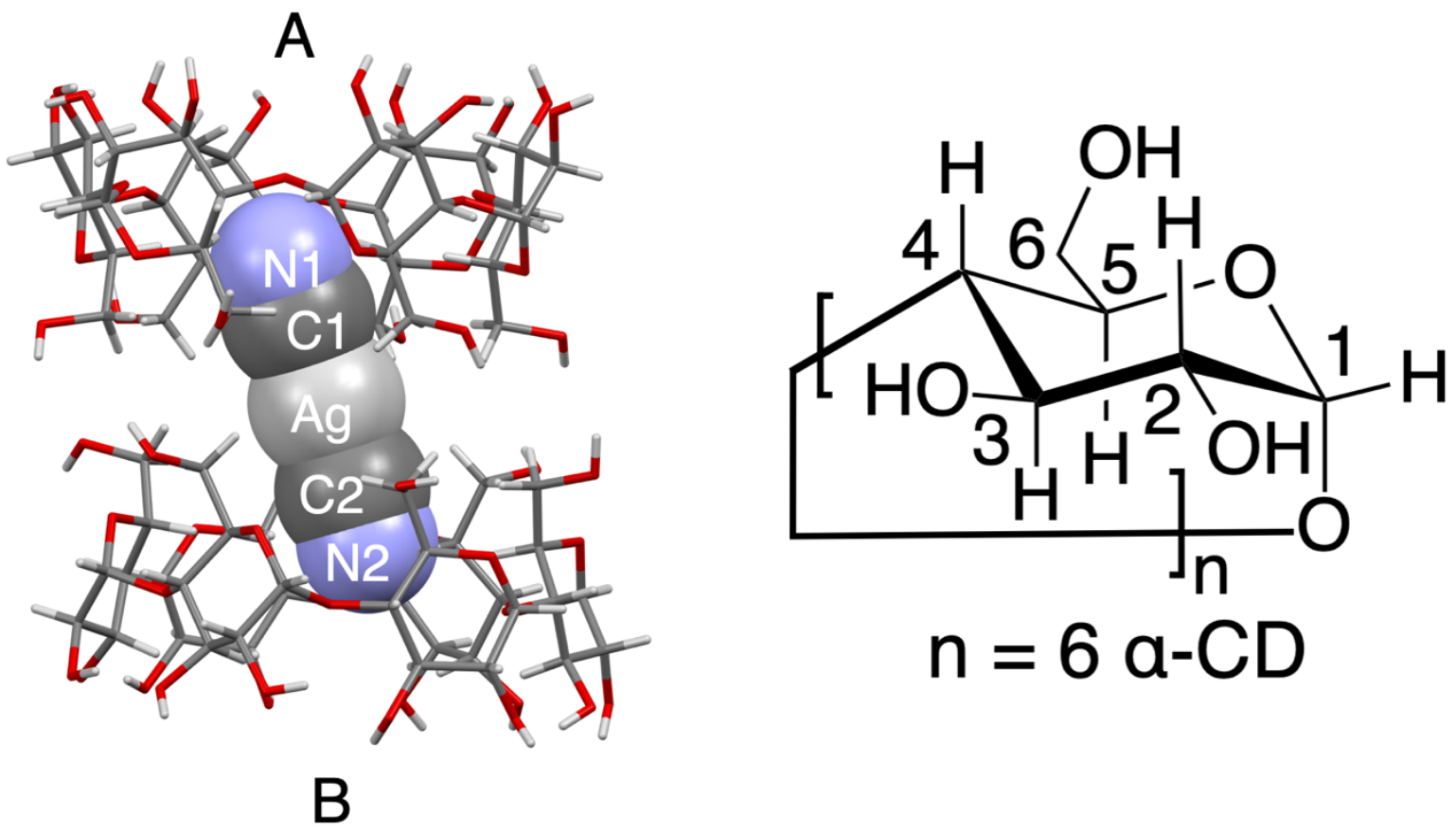

Figure S6. Labeling for $\operatorname{Ag}(\mathrm{CN})_{2}^{-}$and $\alpha$-cyclodextrin in the solid-state superstructure of $\mathrm{KAg}(\mathrm{CN})_{2} \subset(\alpha-\mathrm{CD})_{2}$. The top $\alpha-\mathrm{CD}$ is labeled as $\mathrm{A}$ and the bottom $\alpha-\mathrm{CD}$ is labeled as B

Table S3. Distances between $\operatorname{Ag}(\mathrm{CN})_{2}{ }^{-}$and the inner protons $\mathrm{H}-5$ and $\mathrm{H}-6$ in each of the six glucose subunits of the two $\alpha$-CDs (A and B), labels are shown in Figure S6

\begin{tabular}{ccccccc}
\hline Distances $\AA$ & Unit 1 & Unit 2 & Unit 3 & Unit 4 & Unit5 & Unit 6 \\
\hline C1-AH6 & $4.0 / 3.6$ & $4.1 / 4.2$ & $4.2 / 4.4$ & $4.6 / 4.4$ & $4.0 / 3.9$ & $3.9 / 3.6$ \\
Ag-AH6 & $4.1 / 3.7$ & $3.5 / 3.9$ & $3.8 / 4.4$ & $4.8 / 5.1$ & $4.7 / 4.9$ & $4.6 / 4.5$ \\
N1-AH5 & 3.1 & 3.6 & 3.8 & 3.5 & 2.8 & 2.8 \\
C2-BH6 & $3.5 / 4.1$ & $3.5 / 3.9$ & $4.0 / 4.2$ & $4.7 / 5.0$ & $4.7 / 4.4$ & $4.0 / 4.0$ \\
Ag-BH6 & $4.3 / 3.8$ & $4.4 / 4.5$ & $4.9 / 4.7$ & $5.1 / 4.9$ & $4.5 / 3.7$ & $3.7 / 3.4$ \\
N2-BH5 & 2.7 & 2.8 & 3.3 & 3.9 & 3.8 & 3.2 \\
\hline
\end{tabular}




\section{Section 3. Computational Investigations}

\section{Visualization of Noncovalent Interactions}

Independent Gradient Model Analysis Independent gradient model (IGM) analysis is an approach $^{\mathrm{S} 5}$ based on promolecular density (an electron density model prior to molecule formation) to identify and isolate intermolecular interactions. Strong polar attractions and van der Waals contacts are visualized as an iso-surface with blue and green color, respectively. Single crystal superstructures were used as input files. The binding surface was calculated by Multiwfn 3.6 program $^{\mathrm{S} 6}$ through function 20 (visual study of weak interaction) and visualized by Chimera software. ${ }^{\mathrm{S} 7}$

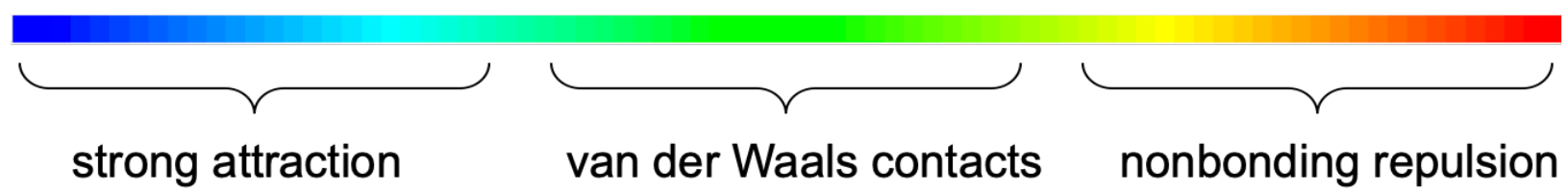

Figure S7. Color-coded sign $\left(\lambda_{2}\right)_{\rho}$ scale bar
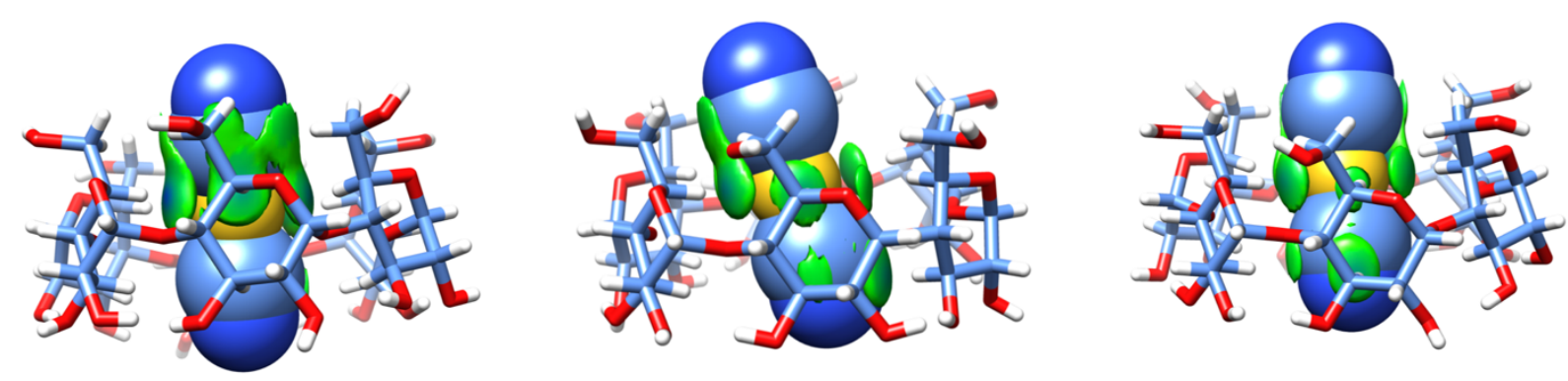

Figure S8. Tubular $(\alpha-\mathrm{CD})$ and space-filling $\left(\mathrm{Au}(\mathrm{CN})_{2}{ }^{-}\right)$representations of $\mathrm{Au}(\mathrm{CN})_{2}{ }^{-} \subset \alpha-\mathrm{CD}$ showing intermolecular binding iso-surface. $\Delta \kappa$ inter $(\rho)=0.003$ a.u. Iso-surfaces are colored according to a BGR scheme over the range $-0.03<\operatorname{sign}\left(\lambda_{2}\right) \rho<+0.05$ a.u. H: white, C: pale blue, $\mathrm{N}$ : blue, O: red and Au: yellow.
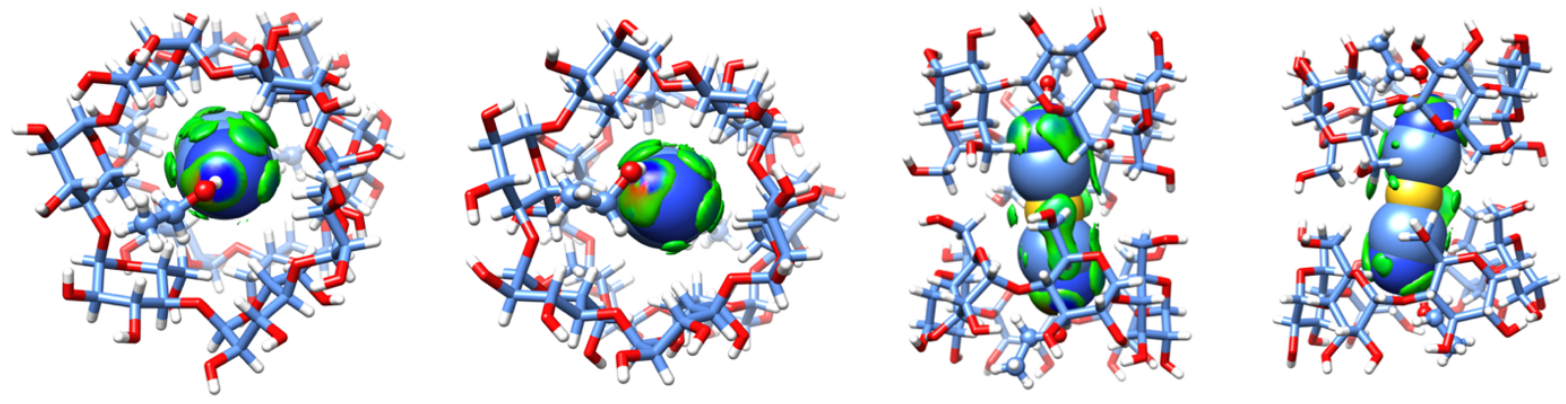

Figure S9. Tubular $(\alpha-\mathrm{CD})$ and space-filling $\left(\mathrm{Au}(\mathrm{CN})_{2}{ }^{-}\right)$representations of $\mathrm{Au}(\mathrm{CN})_{2}{ }^{-} \subset(\alpha-\mathrm{CD})_{2}$ showing tntermolecular binding iso-surface. $\Delta \kappa$ inter $(\rho)=0.003$ a.u. Iso-surfaces are colored according to a BGR scheme over the range $-0.03<\operatorname{sign}\left(\lambda_{2}\right) \rho<+0.05$ a.u. H: white, C: pale blue, $\mathrm{N}$ : blue, O: red and Au: yellow. 

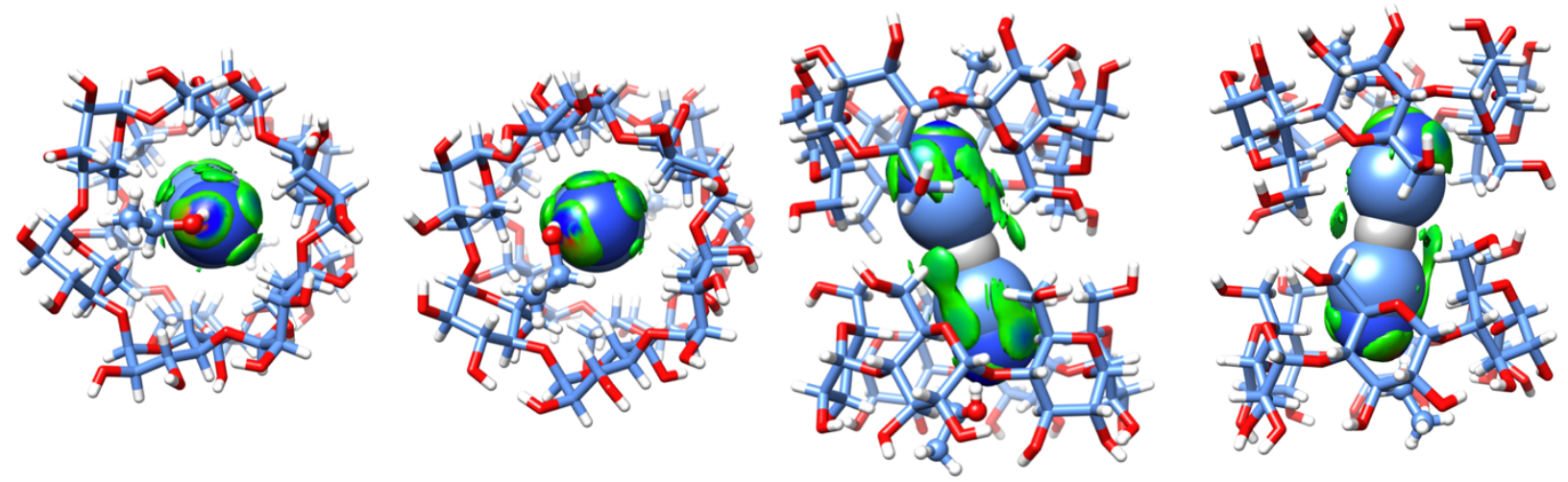

Figure S10. Tubular $(\alpha-\mathrm{CD})$ and space-filling $\left(\mathrm{Ag}(\mathrm{CN})_{2}{ }^{-}\right)$representations of $\mathrm{Ag}(\mathrm{CN})_{2}{ }^{-} \subset(\alpha-\mathrm{CD})_{2}$ showing intermolecular binding iso-surface. $\Delta \kappa$ inter $(\rho)=0.003$ a.u. Iso-surfaces are colored according to a BGR scheme over the range $-0.03<\operatorname{sign}\left(\lambda_{2}\right) \rho<+0.05$ a.u. H: white, C: pale blue, $\mathrm{N}$ : blue, O: red and Au: yellow.

\section{Binding Energy Analysis}

The $x y z$ coordinates for the single-point calculations were extracted from the X-ray single crystal data. All optimizations and single-point calculations were performed with density functional theory (DFT) in the Orca program ${ }^{\mathrm{S} 8}$ (version 4.1.2) using the Coulomb attenuated method (rangecorrected) and hybrid Becke three-parameter Lee-Yang-Parr ${ }^{\mathrm{S} 9}$ (CAM-B3LYP) functional, the Ahlrich's double zeta basis set with a polarization function ${ }^{\mathrm{S} 10}$ Def2-SVP, and Grimme's thirdgeneration dispersion with Beck Johnson damping (D3BJ). In order to speed up the DFT optimizations, the Coulomb integral and numerical chain-of-sphere integration for the HF exchanges $^{\mathrm{S} 11, \mathrm{~S} 12}$ (RIJCOSX) method was applied with the Def2/J auxiliary basiss ${ }^{\mathrm{S} 13}$ (AuxJ). The optimizations in a water continuum were performed with the Conductor like Polarizable Continuum Model ${ }^{\mathrm{S} 14}$ (CPCM) in Orca.

(a)

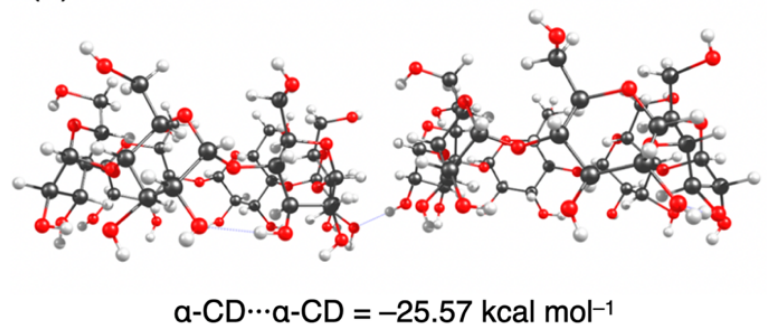

(b)

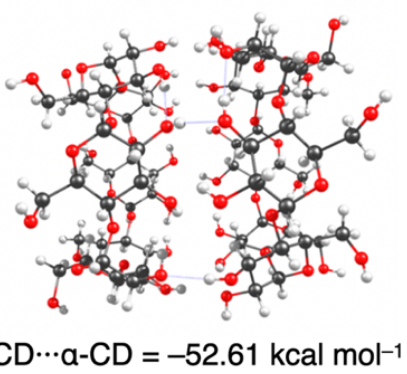

Figure S11. Ball-and-stick representations showing the binding energies of the cyclodextrins of (a) edge-to-edge and (b) face-to-face in the (1:2) crystal 
(a)

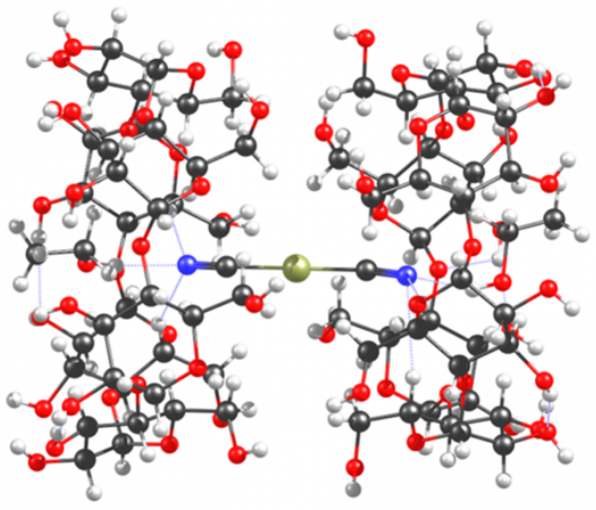

(b)
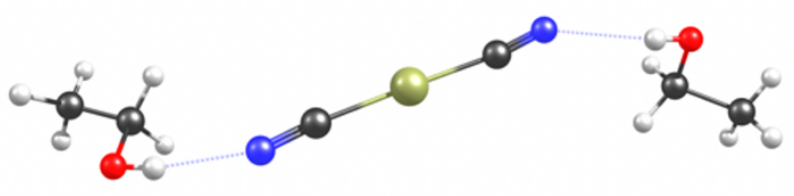

$\mathrm{Au}(\mathrm{CN})_{2}{ }^{-\cdots}(\mathrm{EtOH})_{2}=-22.49 \mathrm{kcal} \mathrm{mol}^{-1}$

Figure S12. Ball-and-stick representations showing the binding energies in the (1:2) crystal between (a) the gold cyanide, the cyclodextrins and the EtOH molecules; (b) just the gold cyanide and the $\mathrm{EtOH}$ molecule

Table S4. Binding energies for optimizations in vacuum of (1:1) structures

\begin{tabular}{|l|l|l|}
\hline Entry & Electronic Energy (DFT) & Binding Energy $\left(\mathrm{kcal} \mathrm{mol}{ }^{-1}\right)$ \\
\hline$\alpha-\mathrm{CD}$ & -3660.606331 & NA \\
\hline $\mathrm{Ag}(\mathrm{CN})_{2}^{-}$ & -332.4985083 & NA \\
\hline $\mathrm{Au}(\mathrm{CN})_{2}^{-}$ & -321.2813003 & NA \\
\hline$\alpha-\mathrm{CD}-\mathrm{Ag}(\mathrm{CN})_{2}^{-}$ & -3993.162487 & -36.17 \\
\hline$\alpha-\mathrm{CD}-\mathrm{Au}(\mathrm{CN})_{2}^{-}$ & -3981.944433 & -35.64 \\
\hline
\end{tabular}

Table S5. Binding energies for optimizations in water continuum of (1:1) structures

\begin{tabular}{|l|l|l|}
\hline Entry & Electronic Energy (DFT) & Binding Energy $\left(\mathrm{kcal} \mathrm{mol}^{-1}\right)$ \\
\hline$\alpha-\mathrm{CD}$ & -3660.647251 & NA \\
\hline $\mathrm{Ag}(\mathrm{CN})_{2}^{-}$ & -332.5811008 & NA \\
\hline $\mathrm{Au}(\mathrm{CN})_{2}^{-}$ & -321.3620598 & NA \\
\hline$\alpha-\mathrm{CD}-\mathrm{Ag}(\mathrm{CN})_{2}^{-}$ & -3993.286396 & -36.42 \\
\hline$\alpha-\mathrm{CD}-\mathrm{Au}(\mathrm{CN})_{2}^{-}$ & -3982.061353 & -32.66 \\
\hline
\end{tabular}


Table S6. Binding energies for (1:1) single crystal with $\mathrm{Au}(\mathrm{CN})_{2}^{-}$

\begin{tabular}{|c|c|c|}
\hline Entry & $\begin{array}{l}\text { Electronic Energy } \\
\text { (DFT) }\end{array}$ & $\begin{array}{l}\text { Binding Energy } \\
\left(\mathrm{kcal} \mathrm{mol}^{-1}\right)\end{array}$ \\
\hline $\mathrm{Au}(\mathrm{CN})_{2}^{-}$ & -321.2455053 & NA \\
\hline $\mathrm{K} 1^{+}$ & -599.6908053 & $\mathrm{NA}$ \\
\hline A & -3659.75888 & NA \\
\hline B & -3659.785735 & NA \\
\hline $\mathrm{C}$ & -3659.808142 & $\mathrm{NA}$ \\
\hline $\mathrm{D}$ & -3659.808339 & NA \\
\hline $\mathrm{E}$ & -3659.785714 & NA \\
\hline $\mathrm{F}$ & -3659.785733 & $\mathrm{NA}$ \\
\hline $\mathrm{G}$ & -3659.808142 & $\mathrm{NA}$ \\
\hline $\mathrm{H}$ & -3659.808266 & NA \\
\hline $\mathrm{I}$ & -3659.785768 & $\mathrm{NA}$ \\
\hline $\mathrm{J}$ & -3659.758687 & $\mathrm{NA}$ \\
\hline $\mathrm{K}$ & -3659.758802 & $\mathrm{NA}$ \\
\hline $\mathrm{L}$ & -3659.758991 & $\mathrm{NA}$ \\
\hline $\mathrm{M}$ & -3659.758801 & $\mathrm{NA}$ \\
\hline $\mathrm{N}$ & -3659.758686 & $\mathrm{NA}$ \\
\hline $\mathrm{O}$ & -3659.758897 & NA \\
\hline$A \cdots B$ & -7319.57024 & -16.08 \\
\hline$A \cdots C$ & -7319.567617 & -0.37 \\
\hline$A \cdots D$ & -7319.57169 & -2.81 \\
\hline$A \cdots E$ & -7319.561925 & -10.88 \\
\hline$A \cdots F$ & -7319.554659 & -6.30 \\
\hline$A \cdots G$ & -7319.567688 & -0.42 \\
\hline $\mathrm{A} \cdots \mathrm{H}$ & -7319.569673 & -1.59 \\
\hline$A \cdots I$ & -7319.56282 & -11.40 \\
\hline $\mathrm{A} \cdots \mathrm{J}$ & -7319.538412 & -13.08 \\
\hline $\mathrm{A} \cdots \mathrm{K}$ & -7319.53853 & -13.08 \\
\hline $\mathrm{A} \cdots \mathrm{L}$ & -7319.533808 & -10.00 \\
\hline $\mathrm{A} \cdots \mathrm{M}$ & -7319.557437 & -24.95 \\
\hline $\mathrm{A} \cdots \mathrm{N}$ & -7319.557323 & -24.95 \\
\hline $\mathrm{A} \cdots \mathrm{O}$ & -7319.533714 & -10.00 \\
\hline $\mathrm{A} \cdots \mathrm{Au}(\mathrm{CN})_{2}^{-}$ & -3981.055943 & -32.35 \\
\hline$\left(\mathrm{H}_{2} \mathrm{O}\right)_{2}\left(\alpha-\mathrm{CD}_{\mathrm{AHB}}\right)_{3}$ & -11132.05969 & NA \\
\hline $\begin{array}{l}\mathrm{K} 1^{+}\left(\mathrm{H}_{2} \mathrm{O}\right)_{2} \\
\left(\alpha-\mathrm{CD}_{\mathrm{AHB}}\right)_{3}\end{array}$ & -11731.93202 & -113.91 \\
\hline
\end{tabular}

The binding analysis of $[\alpha-\mathrm{CD} \cdots \alpha-\mathrm{CD}]$ interaction is illustrated below. The excess of free water molecules around the cyclodextrins and not bound to $\mathrm{K}^{+}$ions were removed. Only one potassium ion (labeled $\mathrm{K}^{+}$) was selected to reduce computational cost 


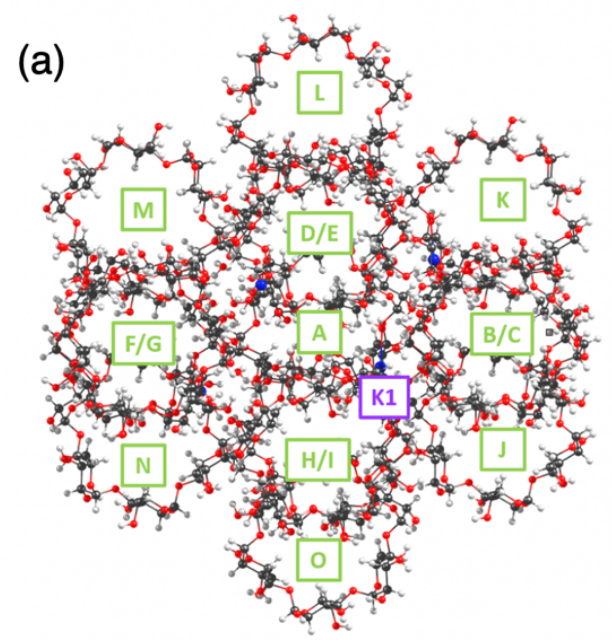

(b)

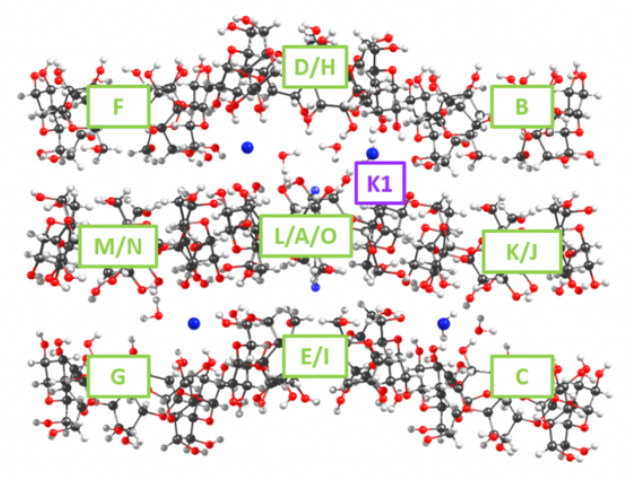

Figure S13. Ball-and-stick representations showing the nearest neighbors of the (1:1) crystal around a central cyclodextrin, which contains a gold cyanide. Each $\alpha-\mathrm{CD}$ and $\mathrm{K}^{+}$is given an alphabetic label and the binding energies between the selected species are presented in Table S6

Table S7. Selected binding energies for $(1: 2)$ single crystal adduct with $\mathrm{Au}(\mathrm{CN})_{2}{ }^{-}$

\begin{tabular}{|c|c|c|}
\hline Entry & $\begin{array}{l}\text { Electronic Energy } \\
\text { (DFT) }\end{array}$ & $\begin{array}{l}\text { Binding Energy } \\
\left(\mathrm{kcal} \mathrm{mol}^{-1}\right)\end{array}$ \\
\hline$\alpha$-CD (left side edge-to-edge) & -3659.805069 & NA \\
\hline$\alpha$-CD (right side edge-to-edge) & -7319.650896 & -25.57 \\
\hline$\alpha$-CD $\cdots \alpha$-CD (edge-to-edge) & -3659.786144 & NA \\
\hline$\alpha$-CD $\cdots \alpha$-CD (edge-to-edge) & -7319.675058 & -52.61 \\
\hline $\mathrm{Au}(\mathrm{CN})_{2}^{-}$ & -321.278387 & NA \\
\hline$\alpha-C D$ & -3659.786129 & NA \\
\hline $\mathrm{Au}(\mathrm{CN})_{2}{ }^{-\cdots} \alpha-\mathrm{CD}$ & -3981.13207 & -42.39 \\
\hline$\alpha-\mathrm{CD} \cdots \mathrm{EtOH}$ & -3814.569737 & NA \\
\hline $\mathrm{Au}(\mathrm{CN})_{2}-\cdots \alpha-\mathrm{CD} \cdots \mathrm{EtOH}$ & -4135.948188 & -62.79 \\
\hline$(\alpha-C D)_{2}$ & -7319.591187 & NA \\
\hline $\mathrm{Au}(\mathrm{CN})_{2}{ }^{-\cdots}(\alpha-\mathrm{CD})_{2}$ & -7640.996099 & -79.39 \\
\hline$(\alpha-\mathrm{CD})_{2} \cdots(\mathrm{EtOH})_{2}$ & -7629.166154 & NA \\
\hline $\mathrm{Au}(\mathrm{CN})_{2}{ }^{-\cdots}(\alpha-\mathrm{CD})_{2} \cdots(\mathrm{EtOH})_{2}$ & -7950.635384 & -119.75 \\
\hline
\end{tabular}


Table S8. Selected binding energies for (1:1) single crystal adduct with $\mathrm{Ag}(\mathrm{CN})_{2}{ }^{-}$

\begin{tabular}{|l|l|l|}
\hline Entry & $\begin{array}{l}\text { Electronic Energy } \\
(\mathrm{DFT})\end{array}$ & $\begin{array}{l}\text { Binding Energy } \\
\left(\mathrm{kcal} \mathrm{\textrm {mol } ^ { - 1 }}\right)\end{array}$ \\
\hline $\mathrm{Ag}(\mathrm{CN})_{2}^{-}$ & -332.4620557 & NA \\
\hline $\mathrm{A}$ & -3659.75888 & NA \\
\hline $\mathrm{A} \cdots \mathrm{Ag}(\mathrm{CN})_{2}^{-}$ & -3992.270807 & -31.29 \\
\hline
\end{tabular}

Table S9. Selected binding energies for (1:2) single crystal adduct with $\mathrm{Ag}(\mathrm{CN})_{2}^{-}$.

\begin{tabular}{|l|l|l|}
\hline Entry & $\begin{array}{l}\text { Electronic Energy } \\
(\mathrm{DFT})\end{array}$ & $\begin{array}{l}\text { Binding Energy } \\
\left(\mathrm{kcal} \mathrm{mol}^{-1}\right)\end{array}$ \\
\hline $\mathrm{Ag}(\mathrm{CN})_{2}{ }^{-}$ & -332.493334 & NA \\
\hline$\alpha-\mathrm{CD}$ & -3659.780104 & NA \\
\hline $\mathrm{Ag}(\mathrm{CN})_{2}{ }^{-\cdots} \alpha-\mathrm{CD}$ & -3992.323065 & -31.14 \\
\hline $\mathrm{EtOH}$ & -154.7830196 & NA \\
\hline $\mathrm{Ag}(\mathrm{CN})_{2}{ }^{-\cdots} \alpha-\mathrm{CD} \cdots \mathrm{EtOH}$ & -4147.136268 & -50.08 \\
\hline$(\alpha-\mathrm{CD})_{2}$ & -7319.534601 & NA \\
\hline $\mathrm{Ag}(\mathrm{CN})_{2}{ }^{-\cdots}(\alpha-\mathrm{CD})_{2}$ & -7652.147928 & -75.30 \\
\hline$(\alpha-\mathrm{CD})_{2} \cdots(\mathrm{EtOH})_{2}$ & -7629.105661 & NA \\
\hline $\mathrm{Ag}(\mathrm{CN})_{2}{ }^{-\cdots}(\alpha-\mathrm{CD})_{2} \cdots(\mathrm{EtOH})_{2}$ & -7961.779314 & -113.15 \\
\hline
\end{tabular}

Cartesian Coordinates

Optimized in vacuum $(1: 1)$ for $\mathrm{Au}(\mathrm{CN})_{2}{ }^{-} \subset \alpha-\mathrm{CD}$
H 20.15191903913783
0.69841152226427
$-0.58767897402036$
H 19.02243796585125
$-1.42865166542006$
$-0.18491674186483$
H 22.39239578712737
$-0.01567842384358$
$-0.21540509101894$
O 21.66594776067956
$-0.65702108496296$
$-0.19000402497197$
C 20.54183658075170
0.07909125669880
0.24140652312493
H 16.62761748684306
1.35078835932778
1.24005916586200
O 18.38265278670692
$-5.20760899708509$
0.13557512886771
C 19.42320659769979
$-0.87396850534899$
0.68027927659910
H 20.16269568489925
$-3.35943284127273$
H 22.38894283849570
$-2.42428681752956$
0.37717113462929
O 21.86649974465753
1.92921447917924
0.57430424989481
O 18.35819586949036
$-0.13138045466694$
H 18.88436764288902
$-5.85727478468729$
0.85179269579857
H 19.22792031222694
2.33688847478712
1.21042227968183
O 16.70490052696384
1.90983566681529
0.64933786966378
1.21507909623573
2.02482747582271 


\begin{tabular}{|c|c|c|c|}
\hline & 22.46994985437821 & -3.30605517943016 & \\
\hline & 20.93523652317647 & 1.01262154506322 & 1.37070016697586 \\
\hline & 17.48604454200248 & -3.42741783734282 & \\
\hline & 2.17329530444256 & & \\
\hline & 0.11325631801768 & -3.11703780971583 & 517744 \\
\hline & 9.89624634435957 & & \\
\hline & 7.87480767160153 & -4.30647355012883 & \\
\hline & 9.70761618410530 & 1.68267416 & \\
\hline & 7373 & -5.4625 & \\
\hline & 4318 & 4.17395 & \\
\hline & & & \\
\hline & 17.418 & 527 & \\
\hline & 21.3 & & \\
\hline & $21.52674 \xi$ & -3.3576 & \\
\hline & 17.0 & & \\
\hline & 16.82558 & & \\
\hline & 18.944560837 & -3.89 & \\
\hline & 21.7 & -4.6 & \\
\hline & 23.0 & -3.6 & \\
\hline C & 20.0 & & \\
\hline & 19.1 & -0.0 & \\
\hline ) & 19.3 & & \\
\hline & 20.12 & & \\
\hline $\mathrm{O}$ & 22.43 & 3.9 & \\
\hline I & 17.6 & & \\
\hline $\mathrm{H}$ & 21.6 & -2.5 & \\
\hline $\mathrm{O}$ & 22.77 & & \\
\hline & 18.498 & -3.24 & \\
\hline ) & & & \\
\hline & 21.28 & 5.5 & \\
\hline & 23.220 & 4.0 & 298 \\
\hline $\mathrm{C}$ & 21.34741 & & \\
\hline C & & & \\
\hline $\mathrm{H}$ & 20.52654 & & 117 \\
\hline & 17.55532 & & \\
\hline $\mathrm{O}$ & 20.150 & -3.9 & 4.478756 \\
\hline & & & \\
\hline $\mathrm{C}$ & 19.04796 & 3.8560 & 5.193688 \\
\hline & 19.14796 & & \\
\hline $\mathrm{N}$ & $23.143109182^{\prime}$ & -1.8076 & 4.38147502508263 \\
\hline $\mathrm{C}$ & 21.515623189 & 4.0626 & \\
\hline $\mathrm{H}$ & 21.59609549971616 & 2.9611 & \\
\hline $\mathrm{H}$ & 17.65184862530929 & -2.81502078024440 & 4.49078175256309 \\
\hline & 22.30538136687547 & -5.00293299751880 & \\
\hline $\mathrm{O}$ & 17.44172118600318 & 5.60215144520609 & 5.62281149356999 \\
\hline & 17.72952300687356 & 4.24226749785030 & 5.82288412242732 \\
\hline
\end{tabular}




\begin{tabular}{|c|c|c|c|}
\hline & & & \\
\hline & & 4.68373695379782 & \\
\hline & 20.29205059327091 & 4.47034461243843 & 82356325965340 \\
\hline & 20.18755223069179 & & \\
\hline & 20.74797968885006 & -0.17344189870645 & \\
\hline & 19.26633271994479 & -5.35011651214178 & 5.70708913 \\
\hline & .71387223073177 & -4.34386697280500 & 5.75625322 \\
\hline & 1.88933346552803 & & 3152 \\
\hline & 439 & -3.2 & \\
\hline & 819 & -4.5 & \\
\hline & & & \\
\hline & 560 & 0.6 & \\
\hline & & & \\
\hline & 648 & -2.3 & 236 \\
\hline & & & \\
\hline & 20.89 & -4.3 & 9281 \\
\hline & 18.6541915501 & -3.3 & 3065 \\
\hline & & & 727 \\
\hline & 18.1 & & 233 \\
\hline & & & 735 \\
\hline & & -4.9 & 1258 \\
\hline & & & \\
\hline & & & 0117 \\
\hline & & & \\
\hline & & & 499 \\
\hline & 20.4 & -4.7 & 470 \\
\hline & & & 003 \\
\hline & 22.2 & & 0173 \\
\hline & & & 580 \\
\hline & & -1.8 & 728 \\
\hline & 21.6 & & 012 \\
\hline & & & 893 \\
\hline & & & \\
\hline & & & 3537 \\
\hline & 19.2 & -3.8 & \\
\hline & & & 8439 \\
\hline & & & \\
\hline & 16.9 & 630 & 7858 \\
\hline & & & \\
\hline & 19.03 & 3.45 & 544761252636 \\
\hline & 97254293 & 4.48 & 389796539578 \\
\hline & & -1.08 & 9.28501223304703 \\
\hline & 21.56816 & & 9.54277116946779 \\
\hline & & & \\
\hline & 18.43845763218742 & -0.68275670355788 & 9.66531753728311 \\
\hline & 17.83035434413875 & 3.69525470275064 & 10.31731692172025 \\
\hline
\end{tabular}




$\begin{array}{lrrr}\text { H } & 18.78303580697025 & -4.24007213754425 & 9.46231106560664 \\ \text { O } & 20.10171201021029 & 1.40465205338108 & 10.18667312868064 \\ \text { H } & 21.71080381060809 & 5.15171992407781 & 9.97197722755636 \\ \text { C } & 19.37510839095912 & -1.85275745190237 & 9.93754403441997 \\ \text { C } & 20.20888654130293 & 2.81127793329972 & 10.17942611293979 \\ \text { H } & 17.51624888827452 & 2.75710024095235 & 10.80084186703806 \\ \text { H } & 21.59575820915608 & -3.08875623648791 & 10.20050456194585 \\ \text { C } & 20.71090009107214 & -1.37125891268168 & 10.47071780054212 \\ \text { O } & 22.60446258900770 & 2.75604700677123 & 10.43943744816215 \\ \text { O } & 16.35687471951256 & -1.69726376540595 & 10.35652635324271 \\ \text { H } & 18.59236723453222 & 5.35309254300013 & 10.85162039404767 \\ \text { O } & 18.33770149971072 & 0.10409060745260 & 10.86353475835597 \\ \text { H } & 18.90223514765437 & -2.53630486744162 & 10.66313212519028 \\ \text { H } & 22.43368220252977 & 1.87062708602083 & 10.81210908333354 \\ \text { O } & 18.14161998999045 & 4.63395346079890 & 11.31771938923263 \\ \text { O } & 21.51104445239828 & -2.43094334869677 & 10.91927856037687 \\ \text { H } & 20.23665703527562 & 3.20415246785592 & 11.21044500972872 \\ \text { C } & 19.55189712666744 & 0.69397038912101 & 11.23940204811105 \\ \text { H } & 16.46879255860562 & -1.10127446740225 & 11.10949574569408 \\ \text { C } & 20.55326198137523 & -0.39472937058753 & 11.61838453521323 \\ \text { H } & 19.33309429708731 & 1.35163361469473 & 12.09673090624789 \\ \text { O } & 21.79773980177458 & 0.20069154500553 & 11.92978455876507 \\ \text { H } & 22.43649944526131 & -0.52762784461680 & 11.93579161363794 \\ \text { H } & 20.15790474389826 & -0.94227615818347 & 12.49281298705490\end{array}$

\begin{tabular}{ccrc}
\multicolumn{5}{c}{ Optimized in water continuum $(1: 1)$ for $\mathrm{Au}(\mathrm{CN})_{2}{ }^{-} \subset \alpha-\mathrm{CD}$} \\
$\mathrm{H}$ & 20.10281002481319 & 0.73762309310115 & -0.64007084197578 \\
$\mathrm{H}$ & 19.05657431541356 & -1.41584516533251 & -0.13477113407670 \\
$\mathrm{H}$ & 22.35249967718368 & 0.03106778006920 & -0.44591724426230 \\
$\mathrm{O}$ & 21.64335905542999 & -0.59906889398453 & -0.24888692438224 \\
$\mathrm{C}$ & 20.53271426692153 & 0.15495994890572 & 0.19364519281606 \\
$\mathrm{H}$ & 16.55211317796239 & 1.36482366616380 & 1.37925881072434 \\
$\mathrm{O}$ & 18.20402876106542 & -4.82130379839451 & 0.21797352139078 \\
$\mathrm{C}$ & 19.46842592253557 & -0.82355746718220 & 0.69696980965678 \\
$\mathrm{H}$ & 20.21328660559059 & -3.35095588202323 & 0.50414165285163 \\
$\mathrm{H}$ & 22.38351982265795 & -2.23388278209247 & 0.83489119441092 \\
$\mathrm{O}$ & 21.79722612559117 & 2.08639961373142 & 0.74742390953589 \\
$\mathrm{O}$ & 18.39304432261246 & -0.13215503339875 & 1.27215824604851 \\
$\mathrm{H}$ & 18.65686601742748 & -5.57439551666565 & 0.62732698360945 \\
$\mathrm{H}$ & 19.16548811265237 & 2.37522423344768 & 1.19698640540331 \\
O & 16.69767845788512 & 1.90322954822489 & 2.17051621859474 \\
$\mathrm{O}$ & 22.53476062648837 & -3.11233759872364 & 1.23268638905915 \\
$\mathrm{C}$ & 20.93036813945693 & 1.12331178159113 & 1.29417923584701 \\
$\mathrm{H}$ & 17.44321784640681 & -3.03177687550818 & 0.82008174393699 \\
$\mathrm{H}$ & 22.10064987693517 & 2.67765860123407 & 1.46531677434952 \\
$\mathrm{C}$ & 20.16036593756974 & -3.03246883443641 & 1.55741418466884
\end{tabular}




\begin{tabular}{|c|c|c|c|}
\hline & 0.06036560270445 & -1.62609874458845 & \\
\hline & & & \\
\hline & 19.69774872558026 & & \\
\hline & 1.79075264330045 & -5.43593685617364 & \\
\hline & & & \\
\hline & 8.75812777126726 & & \\
\hline & 17.47710549758533 & & \\
\hline & 21.44310341565464 & 0.5411258 & 6012 \\
\hline & & & 5659 \\
\hline & 5890847254 & -4.4 & 7775 \\
\hline & & & 4040 \\
\hline & 12 & -3.6 & 32252 \\
\hline & & & \\
\hline & 23.0976 & & 066 \\
\hline & 20.0155 & & \\
\hline & 19.2 & & \\
\hline & 19.28 & -4.9 & 0024 \\
\hline & & & \\
\hline & 22.3 & & \\
\hline & 17.7 & & \\
\hline & 21.61 & -2.5 & \\
\hline & 22.76 & & \\
\hline[ & & -3.0 & \\
\hline & 18.9 & & \\
\hline I & & & \\
\hline & 23.2 & & \\
\hline & & & \\
\hline & 20.3 & & \\
\hline & 20.4 & & 1451 \\
\hline & & & \\
\hline ) & & & 4. \\
\hline & & & \\
\hline & & & \\
\hline & & & 1309 \\
\hline & & -2.0 & 8404 \\
\hline & 5481962803 & & 78304 \\
\hline & 0871402469 & & 80065 \\
\hline [ & 17.6 & & \\
\hline & 62329346684 & & 86059 \\
\hline ) & 17.5 & & 43955 \\
\hline & 17.72 & & 204524 \\
\hline & 22.69 & -1.3641 & \\
\hline $\mathrm{O}$ & 22.66251018555673 & & 5.39928603586261 \\
\hline & & & \\
\hline & 20.18685074329037 & & 5.78526797948698 \\
\hline & 21.07769307227553 & -0.36789767360569 & 5.6104367150208 \\
\hline
\end{tabular}




\begin{tabular}{|c|c|c|c|}
\hline & 19.29465609886346 & -5.45091480493479 & \\
\hline & 19.72691510528669 & & \\
\hline & & -5.42048104070599 & \\
\hline & 17.38533536804276 & -3.44712473317366 & \\
\hline & & & \\
\hline & 7.74694754708961 & & \\
\hline & 19.40297112710542 & & \\
\hline & 22.75898414295797 & 47339796 & \\
\hline & 206282065483 & & \\
\hline & 21.33080430623578 & 4324667 & \\
\hline & 20.89330 & 27882 & \\
\hline & 18.6391991 & -3.5 & \\
\hline & 105 & & \\
\hline & 18.42154 & & \\
\hline & 20.47062 & 4.0 & \\
\hline & & & \\
\hline & 20.5159 & & \\
\hline & 20.4 & & \\
\hline & 18.229 & -3.9 & \\
\hline & & & \\
\hline & 20.40274 & & \\
\hline & & & \\
\hline & 22.2147 & & \\
\hline & 22.8614 & & \\
\hline & 17.1 & & \\
\hline & 21.6624 & 2.4 & \\
\hline & 174 & & \\
\hline & 18.9435 & -0.1 & \\
\hline & 19.7017 & -2.6 & \\
\hline & 19.23215 & & \\
\hline & 21.42602 & & \\
\hline & & & \\
\hline & 16.9807 & & \\
\hline & 23.598 & 646 & \\
\hline & & & \\
\hline & 21.69358 & 1752 & 8.997 \\
\hline & 17.07800 & & \\
\hline & 21.58056820167551 & & \\
\hline & 21.25281947616346 & -0.9 & \\
\hline & 18.4759 & -0.7 & \\
\hline & 484842 & & 10.108 \\
\hline & & -4.3 & \\
\hline & 20.17011052209105 & & 10.09763265984220 \\
\hline & & & \\
\hline & 19.37115742954058 & -1.89327426641636 & \\
\hline & 20.22943182371360 & 2.74663828389864 & 10.03821309391366 \\
\hline
\end{tabular}


H 17.47018037158382

H 21.51586253007227

C 20.70076167985398

O 22.61093011766396

O 16.36061752012683

H $\quad 18.37806591239710$

O 18.34806678731957

H 18.85232360975644

H 22.41682886698337

O 17.97808441421031

O 21.42186479828424

H 20.23305121349634

C 19.56312438359344

H 16.40294991524861

C 20.53478496301792

H $\quad 19.32565598631456$

O 21.76375583481820

H 22.41168305578837

H 20.11456811414539
2.35354131218322 $-3.19078267071605$ $-1.43285158655151$ 2.71748591207814 $-1.73094688173786$ 4.94016686409487 0.11383569230959 $-2.53096334007385$ 1.83752002477934 4.09251631760760 $-2.52116688956997$ 3.17427896119938 0.68819480196516 $-1.13789843159706$ $-0.39256690278286$ 1.38595523760640 0.24010208902401 $-0.45629718806386$ $-0.88111353501248$
10.37909196841878 10.34210684170269 10.53273362853139 10.31034520019505

10.21046019456202 11.05851795051271 10.75484131483885 10.69388062098972 10.68732169053241 11.30429235840096 11.04985516798609 11.05466126901547 11.15889068702966 10.97475156522095 11.62332750299279 11.97662945979692 11.92381069278499 12.10599159132084 12.51984748325588

Optimized in vacuum (1:1) for $\mathrm{Ag}(\mathrm{CN})_{2}{ }^{-} \subset \alpha-\mathrm{CD}$
H 20.03082431700694
0.61034063389969
H 18.86762202841305
$-1.48192969427335$
$-0.43733728794067$
H 22.26213386998604
$-0.09892913959332$
O 21.54014587928770
$-0.74775096616937$
$-0.06995813387584$
$-0.05728585239219$
$-0.05362600114885$
C 20.41463431085589
$-0.01979263749384$
0.38650342948610
H $\quad 16.53328514187649$
1.33159737904967
O $\quad 18.56554390402676$
$-5.40249727430595$
C 19.27427954914206
$-0.95247599521322$
H 20.09669946817465
$-3.34187915079017$
H 22.29333776148907
$-2.43227706336067$
1.78138018429478
O 21.78481825410790
$-0.17704767503488$
O 18.23074133122532
$-6.01652483442481$
2.26660714395730
H 19.14953741982598
1.89970689201093
O 16.61736760983980
$-3.30736896567041$
C 20.82543744819303
0.90414897721202
1.32786492347105
0.06575875618679
0.80902752335540
0.38835893658668
0.50493433685523
0.98360336828005
1.33016402567883
0.56258928241531
1.34037948914333
2.10545066274546
0.91181210733359
1.51635465403515
$-3.73932263718440$
H 17.48892600037914
2.33493019443378
0.52465215340869
H 22.15144880926167
$-3.18522414773038$
O 19.67449844065309
$-1.84980679895554$
1.70076368095314
1.47755625763226
1.78355352373798
$-4.59280984614816$
1.03767724788345
C 17.95857898575570
1.60904427228304
C 19.61183525822741
$-5.42075037593469$
H 19.92025748469942
4.06810309565244
2.09505578640162
2.11166327141506
2.06261360965429 


\begin{tabular}{|c|c|c|c|}
\hline & & & \\
\hline & 17.29905775777883 & & \\
\hline & 21.26300144193173 & & .32090567558669 \\
\hline & 21.50421232966929 & & \\
\hline & 17.17232455716758 & & \\
\hline & 16.67988672365502 & 0.30875953040982 & \\
\hline & 18.96472432264474 & & \\
\hline & 21.78150699083752 & -4.60802498279016 & 0788 \\
\hline & & & \\
\hline & 20.0842361 & 3.753 & \\
\hline & & & \\
\hline & 19.5 & $-5.2\}$ & 3022 \\
\hline & 20.020 & 2.3 & 2102 \\
\hline & 22.49 & & 223 \\
\hline & 17.53 & 1.79 & 019 \\
\hline & & -2.5 & \\
\hline & 22.8 & -4.4 & 033 \\
\hline & & -3.5 & \\
\hline & $19 .($ & & 870 \\
\hline & & & \\
\hline & 23.27 & & 042 \\
\hline & & & \\
\hline & 20.5 & -5.0 & 493 \\
\hline & 20.6 & -5.9 & 127 \\
\hline I & & & 886 \\
\hline & 20.1 & -4.0 & 797 \\
\hline & & & 513 \\
\hline & 19.0 & & 421 \\
\hline & 19.0 & & 5159 \\
\hline & 23.1 & & 9743 \\
\hline & 21.5 & & \\
\hline & 21.5 & & \\
\hline & 17.6 & -2.9 & 9405 \\
\hline & 22.3 & -4.9 & 0701 \\
\hline & & & 2009 \\
\hline & 17.8 & & 9324 \\
\hline & 22.3 & -0.9 & 3497 \\
\hline $\mathrm{O}$ & 22.808 & & 17141 \\
\hline & 253069313 & & 5850919 \\
\hline H & 20.43 & 5.5 & 065953 \\
\hline & 338411005 & 3194 & 1750949 \\
\hline & 19.33 & -5.3 & 9774712 \\
\hline & 19.74039407231159 & -4.37 & 5.70736011663354 \\
\hline & & & \\
\hline $\mathrm{C}$ & 17.39042869258171 & -3.42435072682787 & 5.37129237990308 \\
\hline & 16.88827606134938 & -4.71348570829349 & 5.12000333401383 \\
\hline
\end{tabular}




\begin{tabular}{|c|c|c|c|}
\hline & 17.88507512781451 & & \\
\hline & & & \\
\hline & 22.86952531833645 & 4.32535468989517 & נים \\
\hline & 9.03974530437374 & & \\
\hline & 1.29548349332255 & & 18200 \\
\hline & & & \\
\hline & 18.64471944322408 & -3.44341041138971 & 71764 \\
\hline & & & 65173 \\
\hline & 18.108776582224 & & \\
\hline & $25-5-1-075077$ & 4.03 & 985 \\
\hline & & & \\
\hline & 20.713 & 5.9 & 2558 \\
\hline & & & \\
\hline & 18.253 & -3.90 & 8296 \\
\hline & & & \\
\hline & 20.4592 & -4.66 & 9109 \\
\hline & & & \\
\hline & 22.303 & -4.8 & \\
\hline & 22.982 & 4.6 & 074 \\
\hline & 17.085 & -1.7 & \\
\hline & 21.61 & 2.4 & 5477 \\
\hline & & & \\
\hline & & -0.0 & 538 \\
\hline & & -2.5 & 4680 \\
\hline & 19.256 & & 3154 \\
\hline & & -4.5 & 009 \\
\hline & & & 895 \\
\hline & 17.00 & & 259 \\
\hline & & & 456 \\
\hline & 0 & & 6241 \\
\hline & 313 & & 9404 \\
\hline & & & 2805 \\
\hline & & & \\
\hline & & & 0516 \\
\hline & & -0.6 & 6926 \\
\hline & & & 2007 \\
\hline & & & 8710 \\
\hline & 20.012 & & 25264 \\
\hline & & & \\
\hline & 19.340 & -1.80 & 20694662 \\
\hline & 2828700731 & & 371286334 \\
\hline & & & 6486647891 \\
\hline & 21.57596933482672 & -3.02240800858973 & 10.14904106901592 \\
\hline & 20.66110691694751 & -1.31631332420293 & \\
\hline & 22.56842454257540 & 2.75113388245361 & 10.49106605543152 \\
\hline & 16.31150516672004 & -1.67299573556618 & 10.17560087946665 \\
\hline
\end{tabular}




$\begin{array}{lrrr}\mathrm{H} & 18.60337131319128 & 5.46020300695483 & 10.85323942345709 \\ \mathrm{O} & 18.26475261495323 & 0.12635606955422 & 10.76238420186451 \\ \mathrm{H} & 18.85587813563757 & -2.49049013451604 & 10.56067716838268 \\ \mathrm{H} & 22.36638392397059 & 1.86821867535714 & 10.85589711093620 \\ \mathrm{O} & 18.12320394976286 & 4.74965780310573 & 11.30291382266788 \\ \mathrm{O} & 21.46414648882843 & -2.36718828069995 & 10.86671686996907 \\ \mathrm{H} & 20.18898286653631 & 3.22709080558931 & 11.22593796831879 \\ \mathrm{C} & 19.45922676090639 & 0.73131559209151 & 11.17027162496025 \\ \mathrm{H} & 16.40005552485643 & -1.08478467132280 & 10.93763912810604 \\ \mathrm{C} & 20.47155533593589 & -0.34657902735115 & 11.55224398301233 \\ \mathrm{H} & 19.21133844370469 & 1.37223238845942 & 12.03232469896932 \\ \mathrm{O} & 21.70515150399271 & 0.26346385796442 & 11.88312767638128 \\ \mathrm{H} & 22.35150704977912 & -0.45811048255777 & 11.90165256866452 \\ \mathrm{H} & 20.07645020799451 & -0.90598091257521 & 12.41873183670094\end{array}$

Optimized in water continuum (1:1) for $\mathrm{Ag}(\mathrm{CN})_{2}{ }^{-} \subset \alpha-\mathrm{CD}$

$\begin{array}{llrl}\text { H } & 20.21765825228998 & 0.77828326002033 & -0.76356949972351 \\ \text { H } & 19.39793523009997 & -1.46792142183770 & -0.24403245007804 \\ \text { H } & 22.53791654179278 & 0.30942077434135 & -0.49822024189536 \\ \text { O } & 21.88582879995283 & -0.39028384196664 & -0.34386747597364 \\ \text { C } & 20.70219992051586 & 0.24743847815811 & 0.07592050438714 \\ \text { H } & 16.62410072919020 & 1.16934317170772 & 1.09924618500166 \\ \text { O } & 18.19190729112313 & -4.37699777522368 & 0.19070270362385 \\ \text { C } & 19.73697514080563 & -0.82184955709169 & 0.58053160397321 \\ \text { H } & 20.45708289242132 & -3.38886503107996 & 0.60469065172117 \\ \text { H } & 22.65688252549783 & -2.24887524965123 & 1.06971912095604 \\ \text { O } & 21.75825568889607 & 2.29304556227511 & 0.60546813682981 \\ \text { O } & 18.58467462971332 & -0.22698039345626 & 1.12076462797655 \\ \text { H } & 18.55231902308697 & -5.21358747967638 & 0.52047657109595 \\ \text { H } & 19.14428922675754 & 2.38054429065548 & 1.08582513496095 \\ \text { O } & 16.71197439479819 & 1.72049410948515 & 1.89009039864498 \\ \text { O } & 22.75024355216517 & -3.12005205534792 & 1.49250865779854 \\ \text { C } & 20.99221646233991 & 1.25798209746262 & 1.16950131807729 \\ \text { H } & 17.61529243025194 & -2.58157018635087 & 0.95717379524449 \\ \text { H } & 22.04999614452347 & 2.89668996115821 & 1.31910556163058 \\ \text { C } & 20.37055368078062 & -2.95402412885945 & 1.61457774196203 \\ \text { O } & 20.40703812681428 & -1.54357867292593 & 1.55305754346000 \\ \text { C } & 17.90549801371021 & -3.58017814831590 & 1.31496664492855 \\ \text { C } & 19.70912973235164 & 1.76930207127139 & 1.80796200618520 \\ \text { H } & 21.88267555944824 & -5.38941309291436 & 2.29171194384363 \\ \text { H } & 19.67268043352223 & 4.26570612624088 & 1.86310562731322 \\ \text { C } & 18.83501681872140 & 0.60211488572427 & 2.25424875564293 \\ \text { C } & 17.48770854932365 & 1.00228383603349 & 2.81448492887193 \\ \text { H } & 21.56224748880141 & 0.73598869763967 & 1.95989873769705 \\ \text { C } & 21.66023380438947 & -3.25759094643753 & 2.37621661966415 \\ \text { H } & 17.06367868442987 & -3.99585817587868 & 1.90029444762040\end{array}$




\begin{tabular}{|c|c|c|c|}
\hline & & & \\
\hline & & & \\
\hline & 21.69042776989595 & & \\
\hline & 22.85870889326737 & & 45263602018004 \\
\hline & 19.91601153359379 & & \\
\hline & 19.37857550341598 & 0.03063765177757 & \\
\hline & & -4.80 & \\
\hline & 20.06850597294777 & 08120 & 2.92805 \\
\hline & & & 88874 \\
\hline & 7.63929307 & & 28019 \\
\hline & & & \\
\hline & 2708240459 & -4.7 & 35573 \\
\hline & & & \\
\hline & & & \\
\hline & 21.06 & & \\
\hline & & & \\
\hline & 21.20 & & 73505 \\
\hline & & -4.9 & 104 \\
\hline & 20.2 & -5.9 & 87544 \\
\hline & 17.6 & & \\
\hline & 20.12 & & 83827 \\
\hline & & & \\
\hline & & & \\
\hline & 19.0 & & 8972 \\
\hline & & -2.2 & 14621 \\
\hline & 21.4 & & 36891 \\
\hline & & & 19609 \\
\hline & 17.6 & -2.9 & 68043 \\
\hline & 22.1 & & 75163 \\
\hline & & & 34866 \\
\hline & 17.7 & & 96172 \\
\hline & 22.17 & & 5.7478 \\
\hline & 22.6 & & 5.27085 \\
\hline & 20.2 & & 80528 \\
\hline & 20.1 & & 74646801 \\
\hline & 20.4 & -0.4 & 20777277 \\
\hline & & & 29540905 \\
\hline & & & \\
\hline & 21.66 & & 6.52557756795051 \\
\hline & 17.3 & -3.3 & 5.66751302514270 \\
\hline & & -4.5 & 428120 \\
\hline & & & \\
\hline & 18.61310364659898 & 0.47071380027318 & 6.36544865990941 \\
\hline & & & \\
\hline & 19.05712211582138 & -2.54746280259400 & 6.60837748980003 \\
\hline & 21.28133579540627 & -3.69554361152432 & 6.95510633119760 \\
\hline
\end{tabular}




\begin{tabular}{|c|c|c|c|}
\hline & 0.76613679899272 & -4.66715682926330 & \\
\hline & 18.58106992850239 & & \\
\hline & & & 16788941921012 \\
\hline & & & \\
\hline & & & \\
\hline & .46516522257376 & -4.95536253502007 & \\
\hline & & & \\
\hline & .48985799404342 & 307 & \\
\hline & & & \\
\hline & 3269704675 & 36 & \\
\hline & & & 007 \\
\hline & 6338558 & -6.0 & \\
\hline & & & \\
\hline & 2466207 & & \\
\hline & 2190835 & -1.9 & 19 \\
\hline & & & \\
\hline & 735032 & & 81 \\
\hline & & & \\
\hline & & -2.7 & 84 \\
\hline & & & \\
\hline & & & 533 \\
\hline & & & \\
\hline & & & \\
\hline & 23.611 & & \\
\hline & & & \\
\hline & 21.71 & & \\
\hline & & & \\
\hline & 21.6056 & & \\
\hline I & 987830 & -0.8 & \\
\hline & & & \\
\hline & & & \\
\hline & & & \\
\hline & 20.1641 & & \\
\hline $\mathrm{H}$ & 21.708 & & \\
\hline & & -1.9 & $10 .($ \\
\hline & 20.2546 & 2.7 & 480 \\
\hline & & & \\
\hline $\mathrm{H}$ & & & \\
\hline & 20.86194132422309 & 012021508 & \\
\hline ) & $22.641^{\prime}$ & & 10.2 \\
\hline & 150878923 & -1.9 & 10.5 \\
\hline & & & \\
\hline ) & 18.46406945803150 & 0.03371262253255 & 10.84234037140412 \\
\hline & & -2.58 & \\
\hline $\mathrm{H}$ & 22.47144614812360 & & 10.58962710678700 \\
\hline$\Omega$ & 18.00228467301158 & 4.07615270690666 & 11.21724103440740 \\
\hline
\end{tabular}




$\begin{array}{lrrr}\mathrm{O} & 21.73696726753219 & -2.42717800154565 & 10.80454472107000 \\ \mathrm{H} & 20.26363108791976 & 3.13974883416908 & 10.96317920604621 \\ \mathrm{C} & 19.69020682338906 & 0.65628923995289 & 11.11991898365094 \\ \mathrm{H} & 16.56292313499205 & -1.30959596924408 & 11.25793343731317 \\ \mathrm{C} & 20.74282586906217 & -0.38002163838578 & 11.50408604073157 \\ \mathrm{H} & 19.50961951332907 & 1.35965115588993 & 11.94712100470600 \\ \mathrm{O} & 21.96132102734946 & 0.30814875122246 & 11.70967857835824 \\ \mathrm{H} & 22.66029409058726 & -0.35750115271337 & 11.79604422630489 \\ \mathrm{H} & 20.41934556385812 & -0.88830023882229 & 12.42923863160478\end{array}$

\section{Section 4. Binding Studies Using NMR Spectroscopy}

${ }^{1} \mathrm{H}$ NMR Titration experiments in $\mathrm{D}_{2} \mathrm{O}$ were performed at $25{ }^{\circ} \mathrm{C}$. Aliquots from a stock solution containing the appropriate $\mathrm{KAu}(\mathrm{CN})_{2}$ were added sequentially to an NMR tube containing the cyclodextrins, and a ${ }^{1} \mathrm{H}$ NMR spectrum was acquired after each addition. The titration isotherms were fitted to either $1: 1$ or 1:2 receptor-substrate binding model using Thordarson's equations ${ }^{\mathrm{S} 15}$ at http://app.supramolecular.org/bindfit/. All the titrations were independently duplicated (shown below is one set of titration isotherms) and all isotherm fittings were used to calculate the average $K_{\mathrm{a}}$ with standard errors.

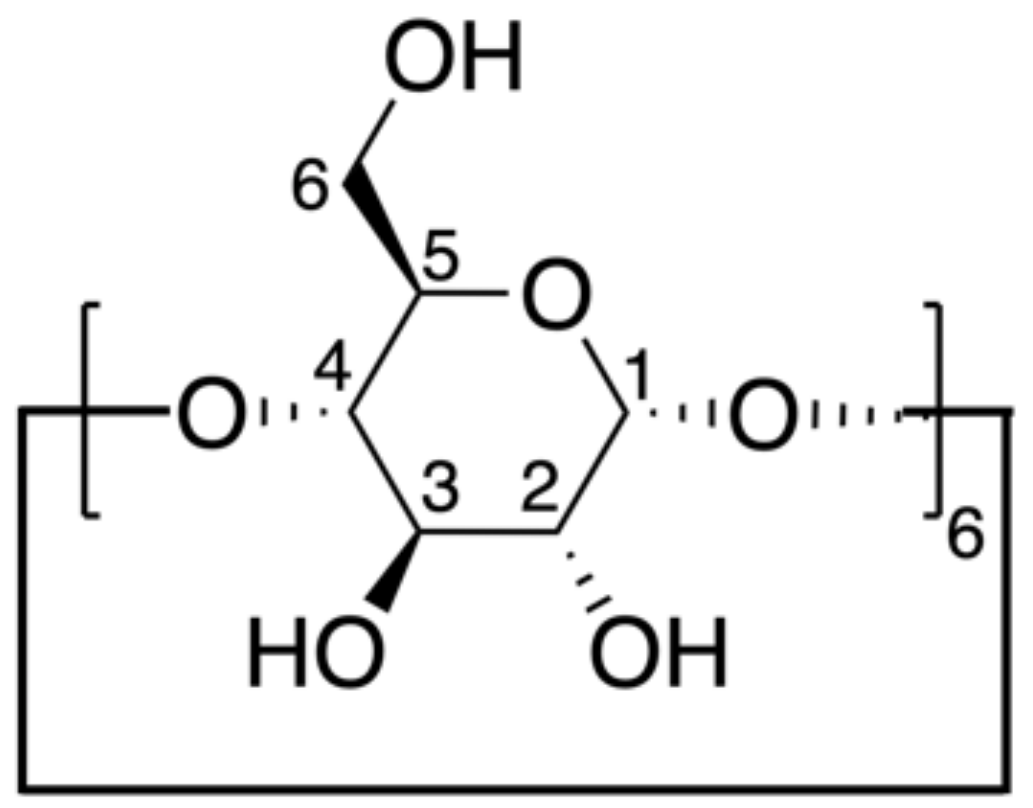

Figure S14. The structural formula for $\alpha-C D$ with labels 


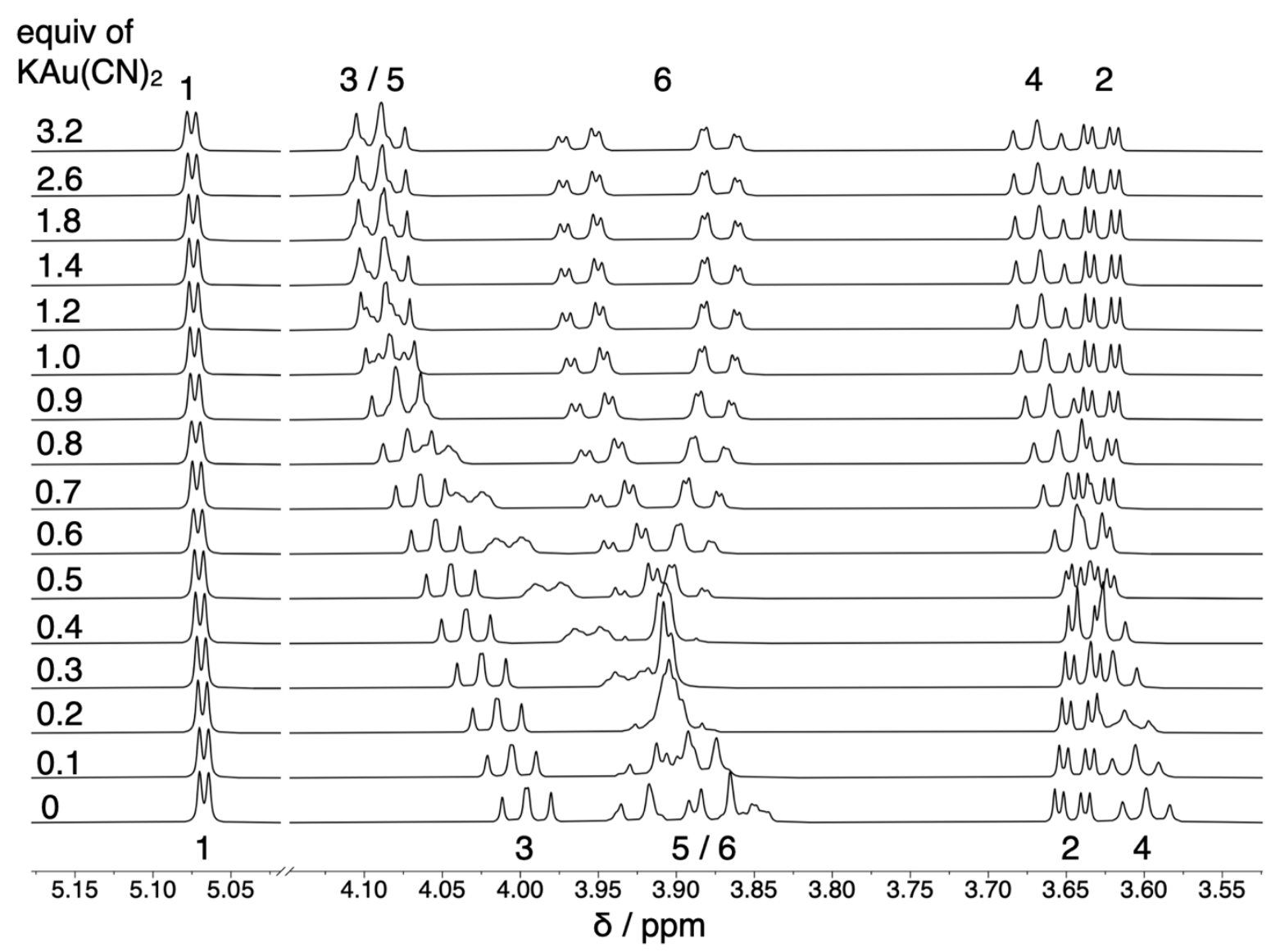

Figure S15. ${ }^{1} \mathrm{H}$ NMR Spectra $\left(600 \mathrm{MHz}, \mathrm{D}_{2} \mathrm{O}, 25{ }^{\circ} \mathrm{C}\right)$ of $\alpha-\mathrm{CD}(5 \mathrm{mM})$ titrated with $\mathrm{KAu}(\mathrm{CN})_{2}$
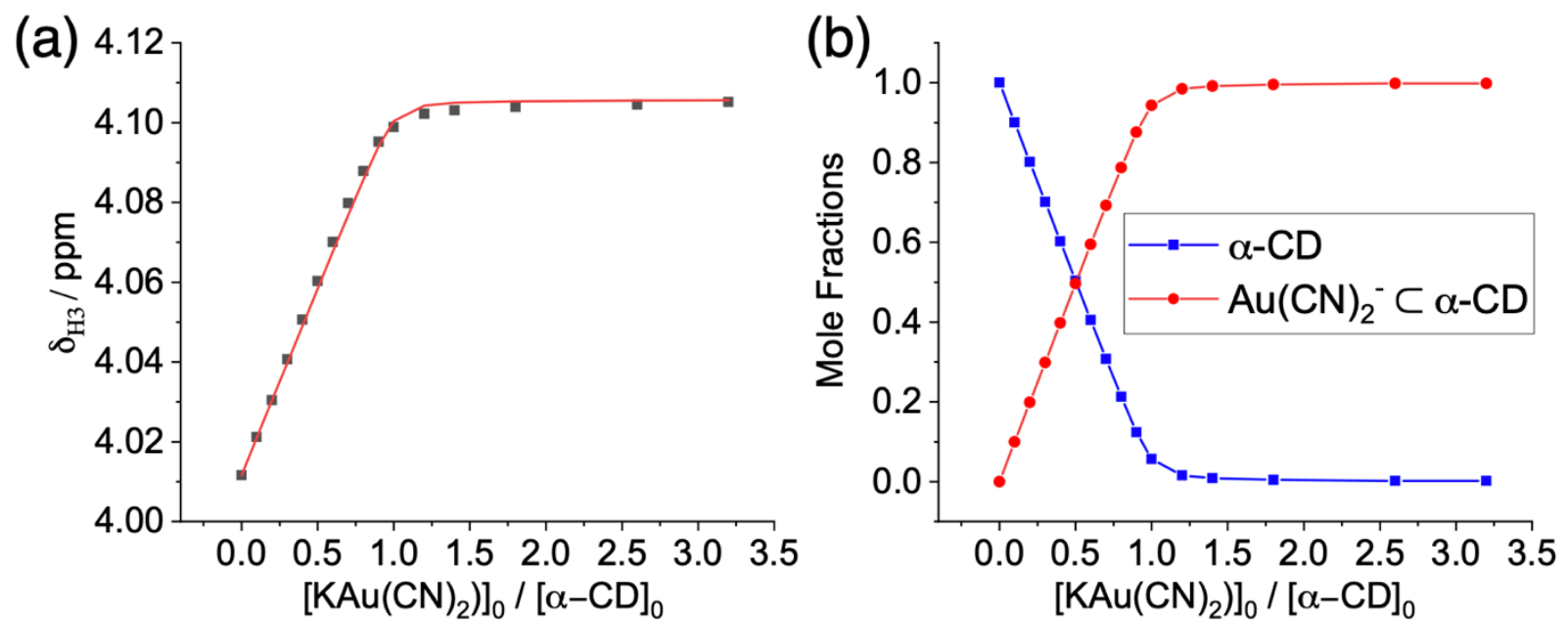

Figure S16. (a) Titration isotherm created by monitoring changes in the chemical shift of $\mathrm{H}-3$ for $\alpha-\mathrm{CD}(5 \mathrm{mM})$ caused by the addition of $\mathrm{KAu}(\mathrm{CN})_{2}$ at $25^{\circ} \mathrm{C}$. Red lines are curve fitting using a 1:1 receptor-substrate binding model. (b) Calculated changes of mole fractions for $\alpha-\mathrm{CD}$ and $\mathrm{Au}(\mathrm{CN})_{2}^{-} \subset \alpha-\mathrm{CD}$ over the substrate-receptor mole ratio 


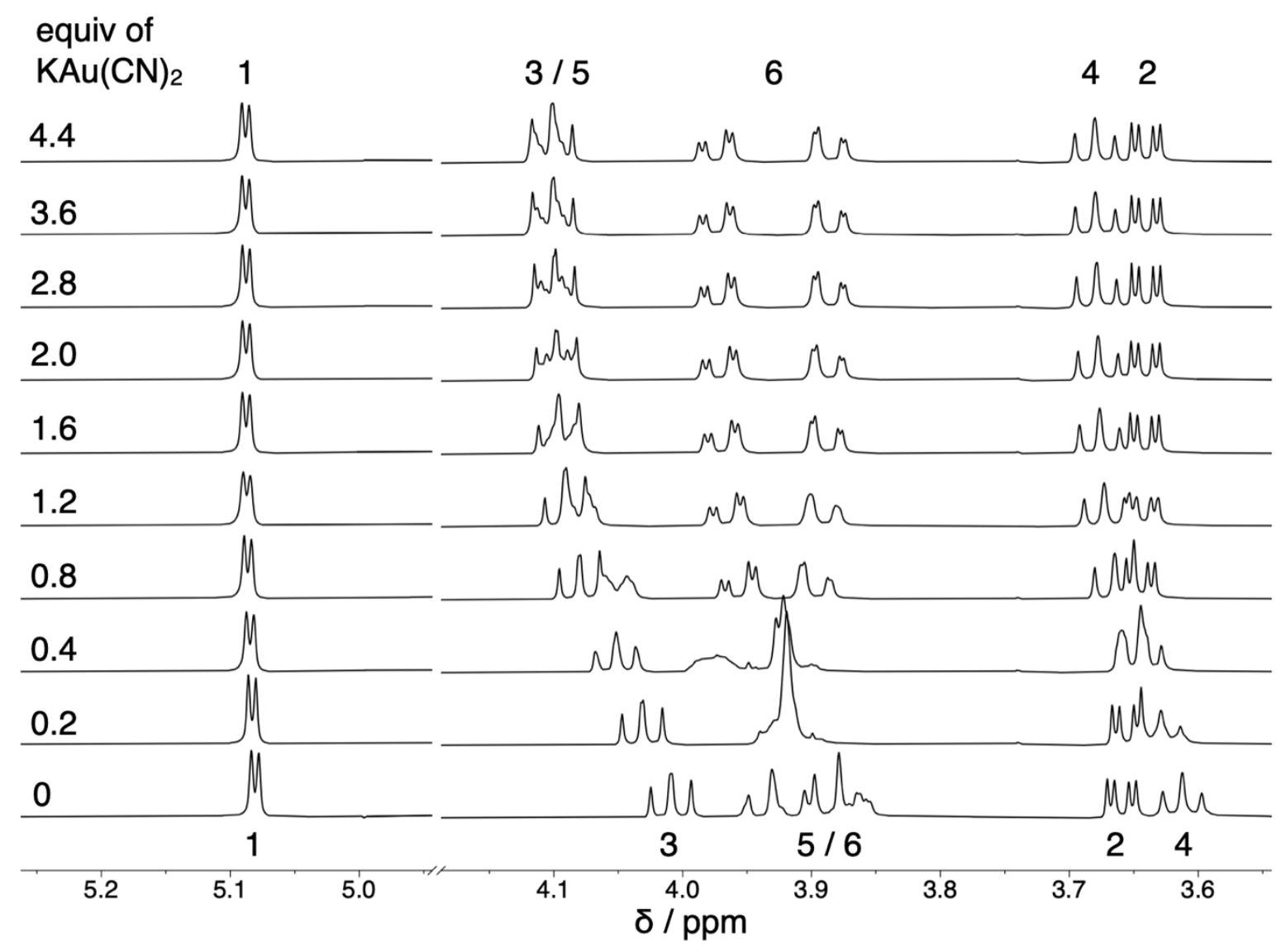

Figure S17. ${ }^{1} \mathrm{H}$ NMR Spectra $\left(600 \mathrm{MHz}, \mathrm{D}_{2} \mathrm{O}, 25{ }^{\circ} \mathrm{C}\right)$ of $\alpha-\mathrm{CD}(0.5 \mathrm{mM})$ titrated with $\mathrm{KAu}(\mathrm{CN})_{2}$
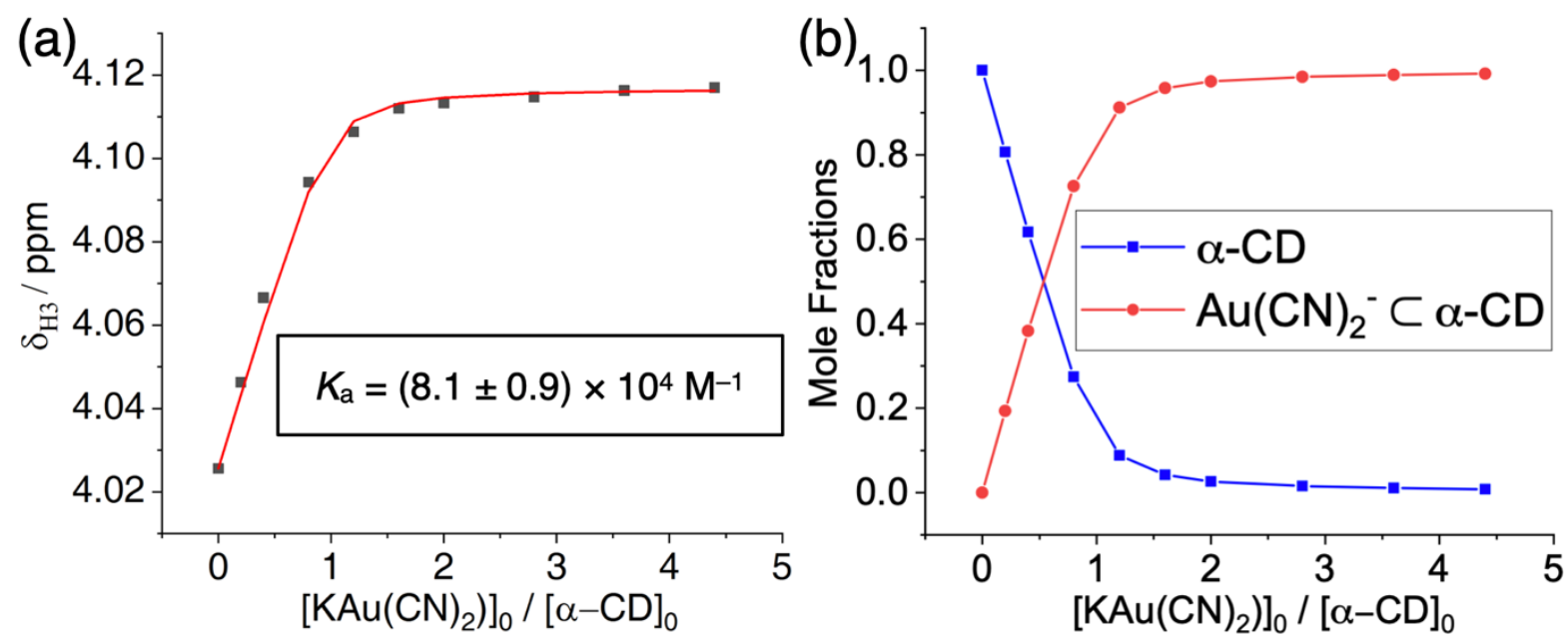

Figure S18. (a) Titration isotherm created by monitoring changes in the chemical shift of $\mathrm{H}-3$ for $\alpha-\mathrm{CD}(0.5 \mathrm{mM})$ caused by the addition of $\mathrm{KAu}(\mathrm{CN})_{2}$ at $25^{\circ} \mathrm{C}$. Red lines are curve fitting using a 1:1 receptor-substrate binding model. (b) Calculated changes of mole fractions for $\alpha-C D$ and $\mathrm{Au}(\mathrm{CN})_{2}^{-} \subset \alpha-\mathrm{CD}$ over the substrate-receptor mole ratio 

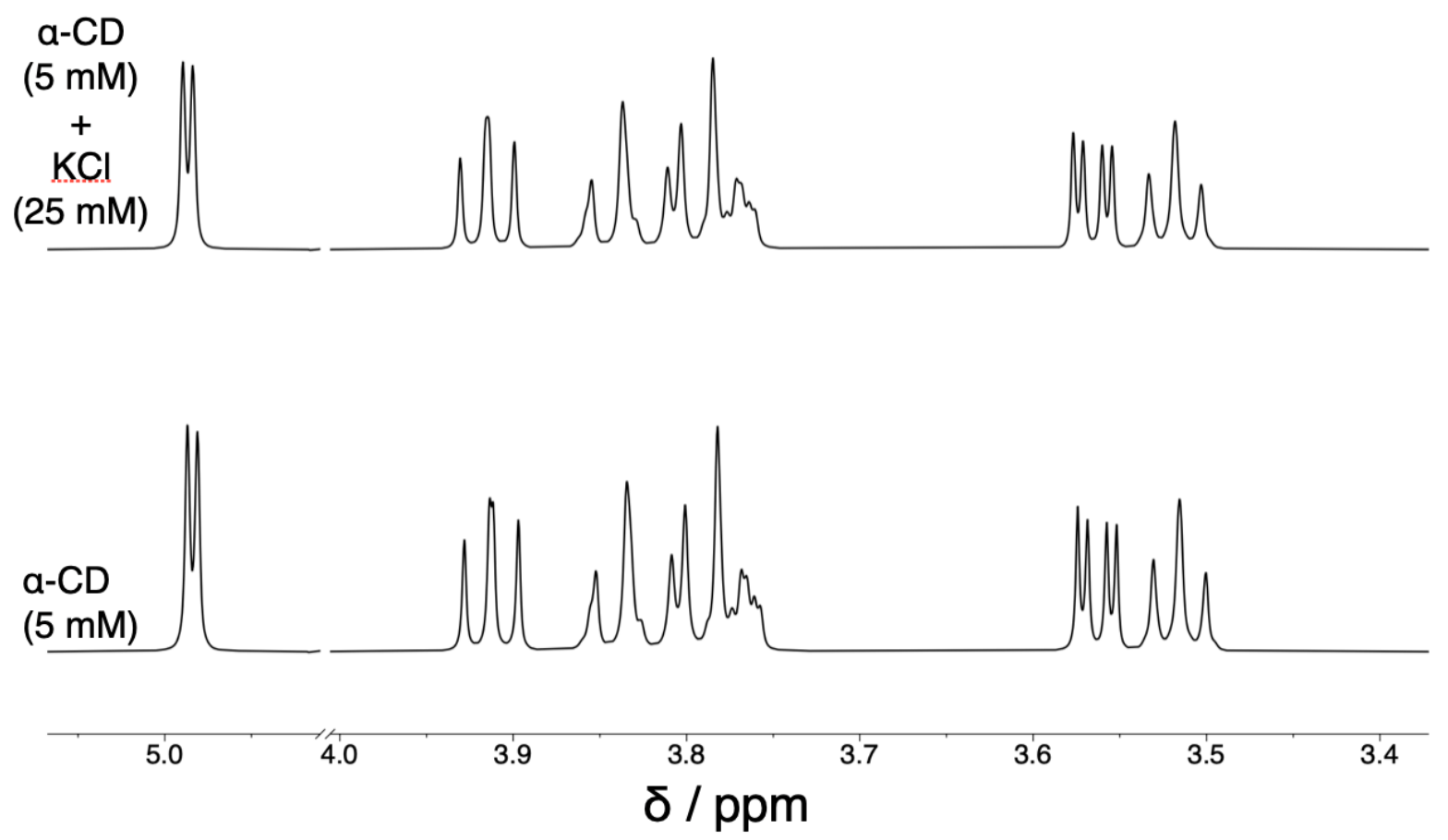

Figure S19. ${ }^{1} \mathrm{H}$ NMR Spectra $\left(600 \mathrm{MHz}, \mathrm{D}_{2} \mathrm{O}, 25{ }^{\circ} \mathrm{C}\right)$ of $\alpha-\mathrm{CD}(5 \mathrm{mM})$ and a mixture of $\alpha-\mathrm{CD}(5$ $\mathrm{mM})$ and $\mathrm{KCl}(25 \mathrm{mM})$

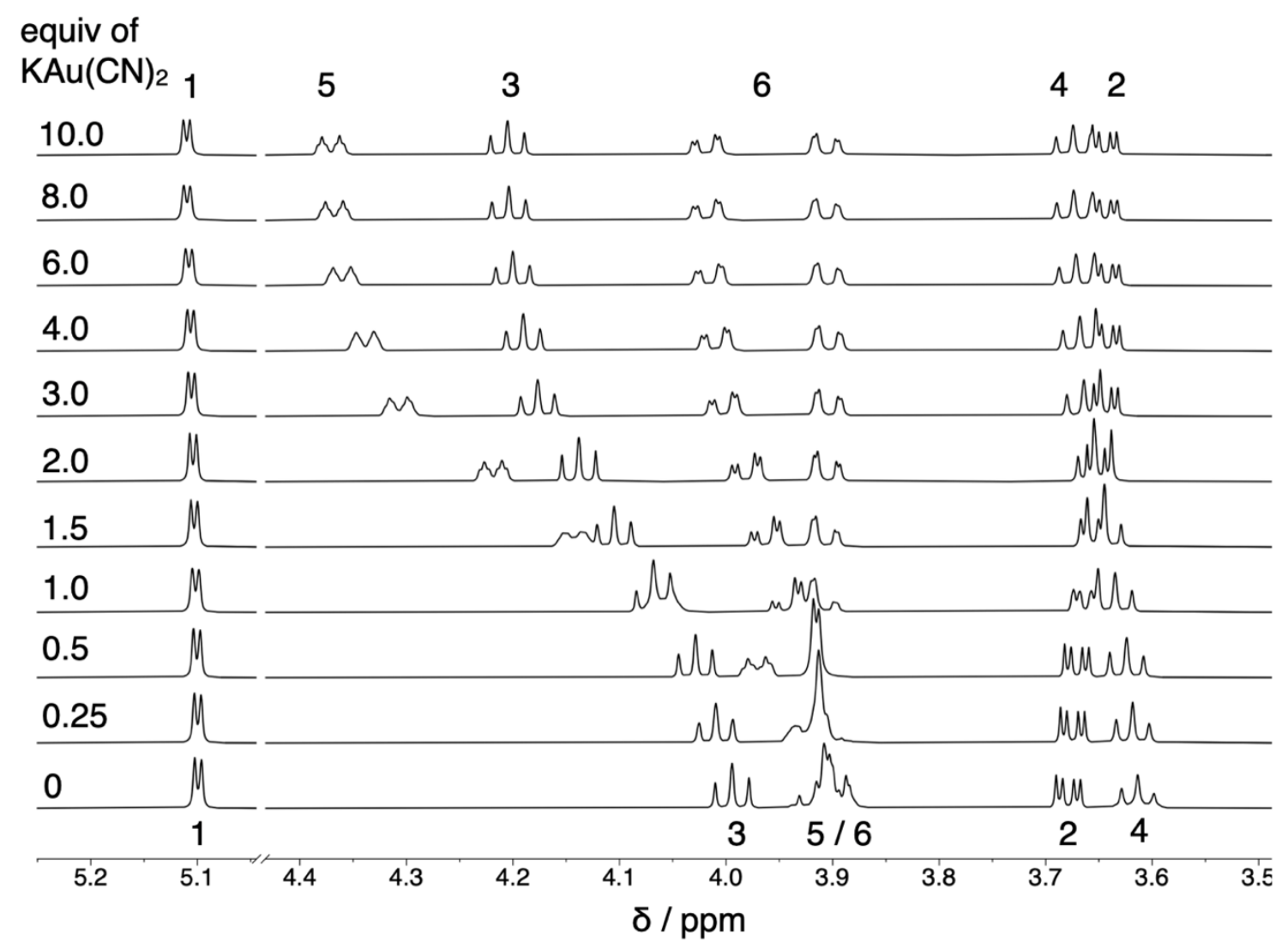

Figure S20. ${ }^{1} \mathrm{H}$ NMR Spectra $\left(600 \mathrm{MHz}, \mathrm{D}_{2} \mathrm{O}, 25{ }^{\circ} \mathrm{C}\right)$ of $\beta-\mathrm{CD}(5 \mathrm{mM})$ titrated with $\mathrm{KAu}(\mathrm{CN})_{2}$ 

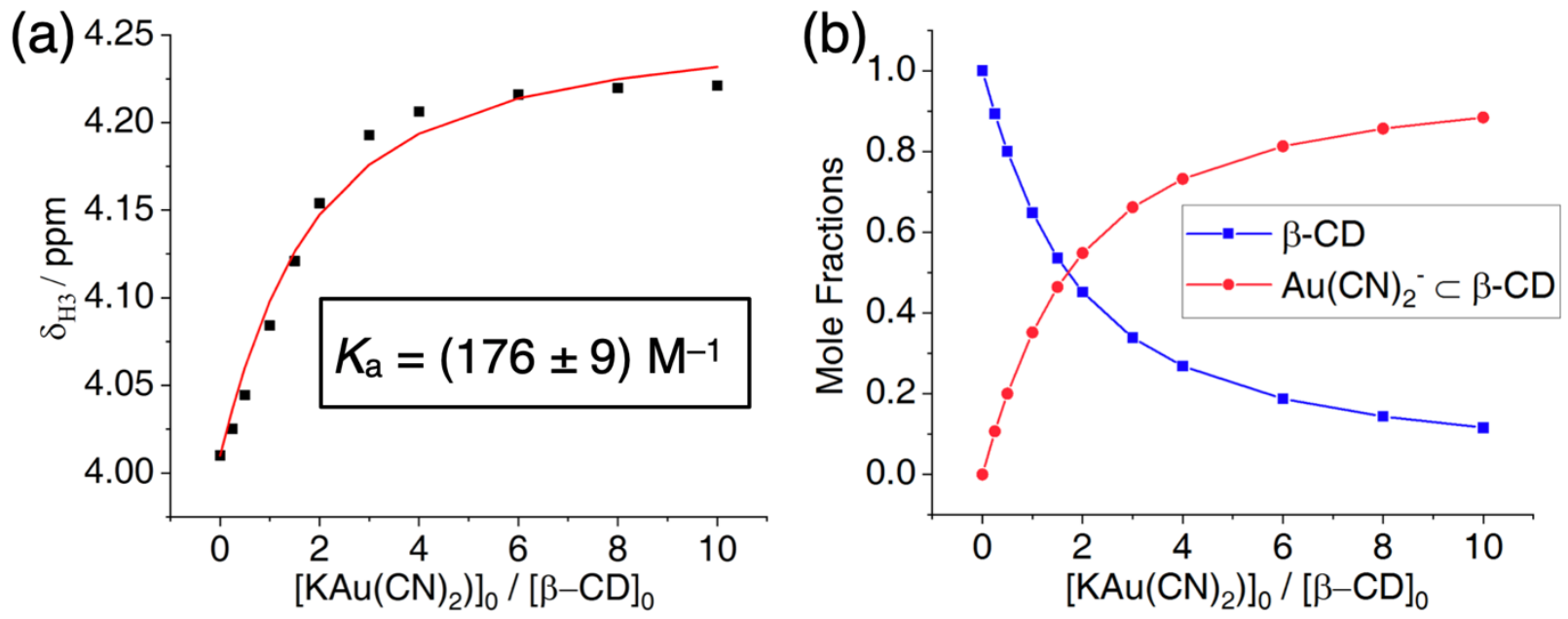

Figure S21. (a) Titration isotherm created by monitoring changes in the chemical shift of $\mathrm{H}-3$ for $\beta-\mathrm{CD}(5 \mathrm{mM})$ caused by the addition of $\mathrm{KAu}(\mathrm{CN})_{2}$ at $25^{\circ} \mathrm{C}$. Red lines are curve fitting using a $1: 1$ receptor-substrate binding model. (b) Calculated changes of mole fractions for $\beta-\mathrm{CD}$ and $\mathrm{Au}(\mathrm{CN})_{2}{ }^{-}$ $\subset \beta$-CD over the substrate-receptor mole ratio
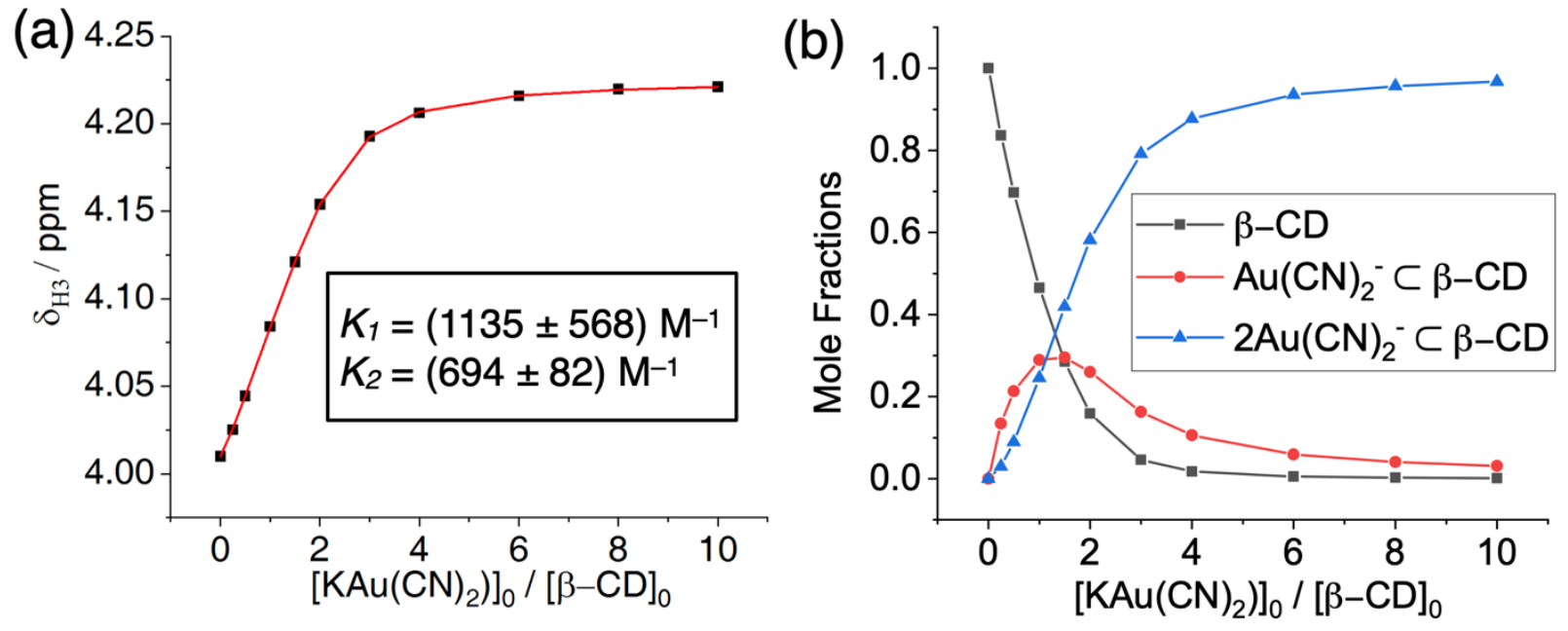

Figure S22. (a) Titration isotherm created by monitoring changes in the chemical shift of $\mathrm{H}-3$ for $\beta-\mathrm{CD}(5 \mathrm{mM})$ caused by the addition of $\mathrm{KAu}(\mathrm{CN})_{2}$ at $25^{\circ} \mathrm{C}$. Red lines are curve fitting using a $1: 2$ receptor-substrate binding model. (b) Calculated changes of mole fractions for $\beta-\mathrm{CD}, \mathrm{Au}(\mathrm{CN})_{2}{ }^{-} \subset$ $\beta-\mathrm{CD}$ and $2 \mathrm{Au}(\mathrm{CN})_{2}^{-} \subset \beta-\mathrm{CD}$ over the substrate-receptor mole ratio 


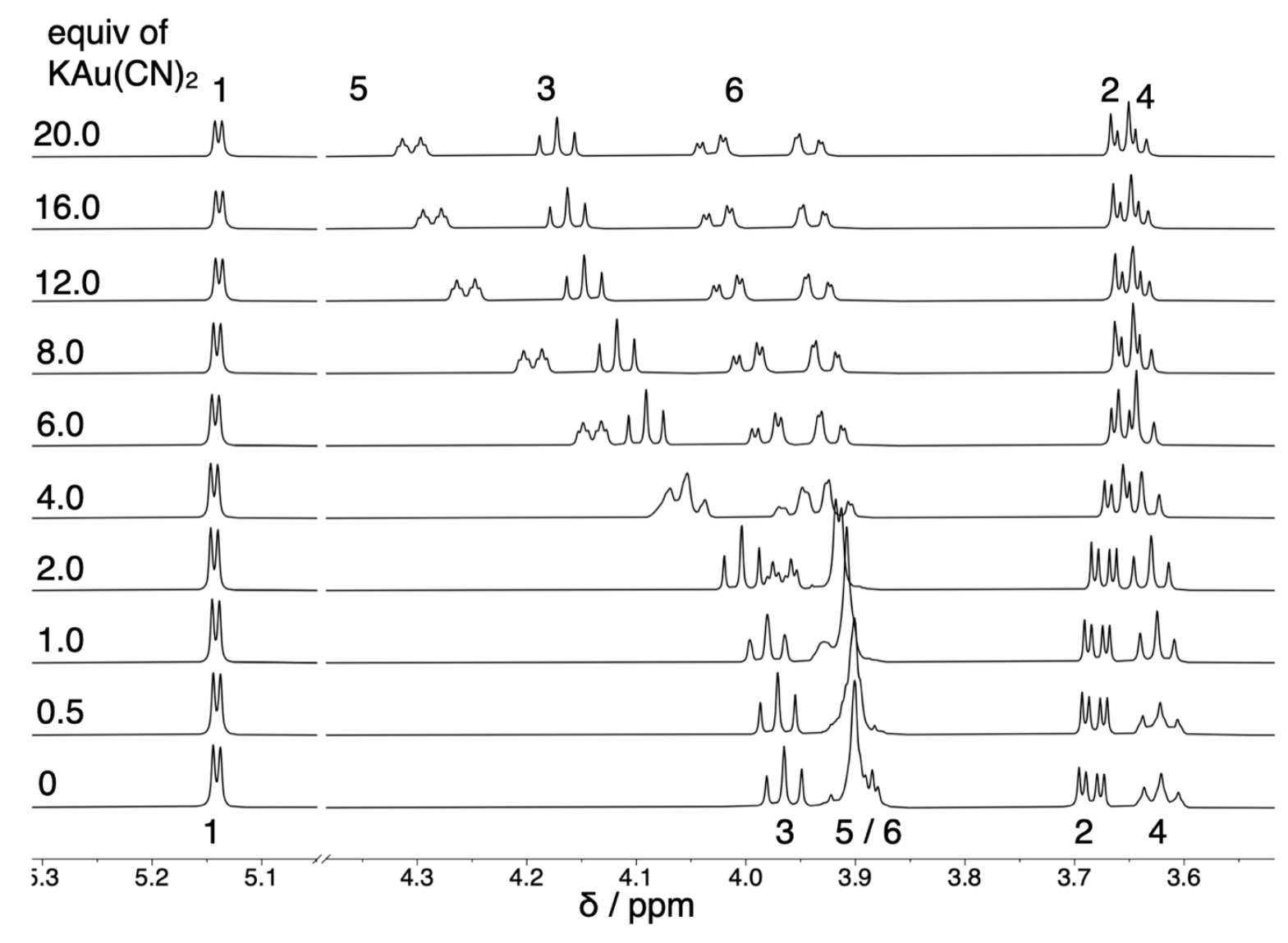

Figure S23. ${ }^{1} \mathrm{H}$ NMR Spectra $\left(600 \mathrm{MHz}, \mathrm{D}_{2} \mathrm{O}, 25{ }^{\circ} \mathrm{C}\right)$ of $\gamma-\mathrm{CD}(5 \mathrm{mM})$ titrated with $\mathrm{KAu}(\mathrm{CN})_{2}$
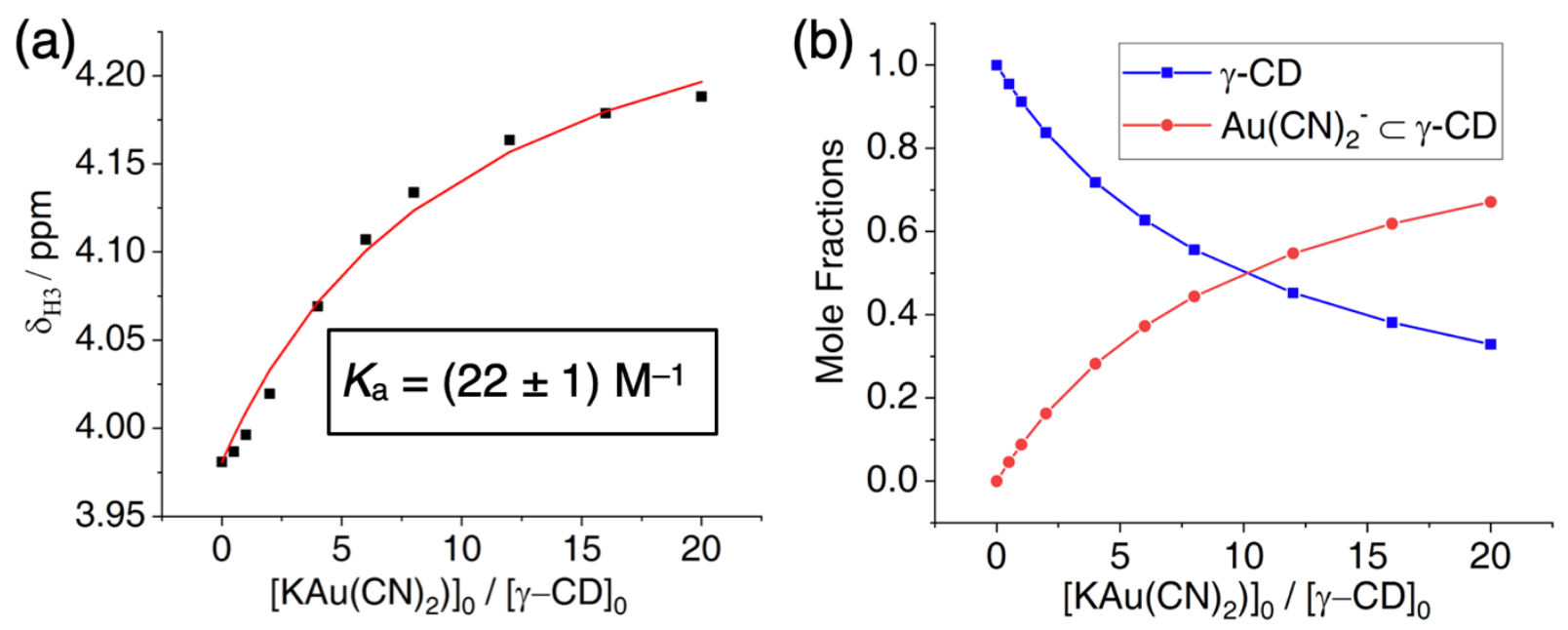

Figure S24. (a) Titration isotherm created by monitoring changes in the chemical shift of $\mathrm{H}-3$ for $\gamma$-CD $(5 \mathrm{mM})$ caused by the addition of $\mathrm{KAu}(\mathrm{CN})_{2}$ at $25^{\circ} \mathrm{C}$. Red lines are curve fitting using a $1: 1$ receptor-substrate binding model. (b) Calculated changes of mole fractions for $\gamma-\mathrm{CD}$ and $\mathrm{Au}(\mathrm{CN})_{2}{ }^{-}$ $\subset \gamma$-CD over the substrate-receptor mole ratio 

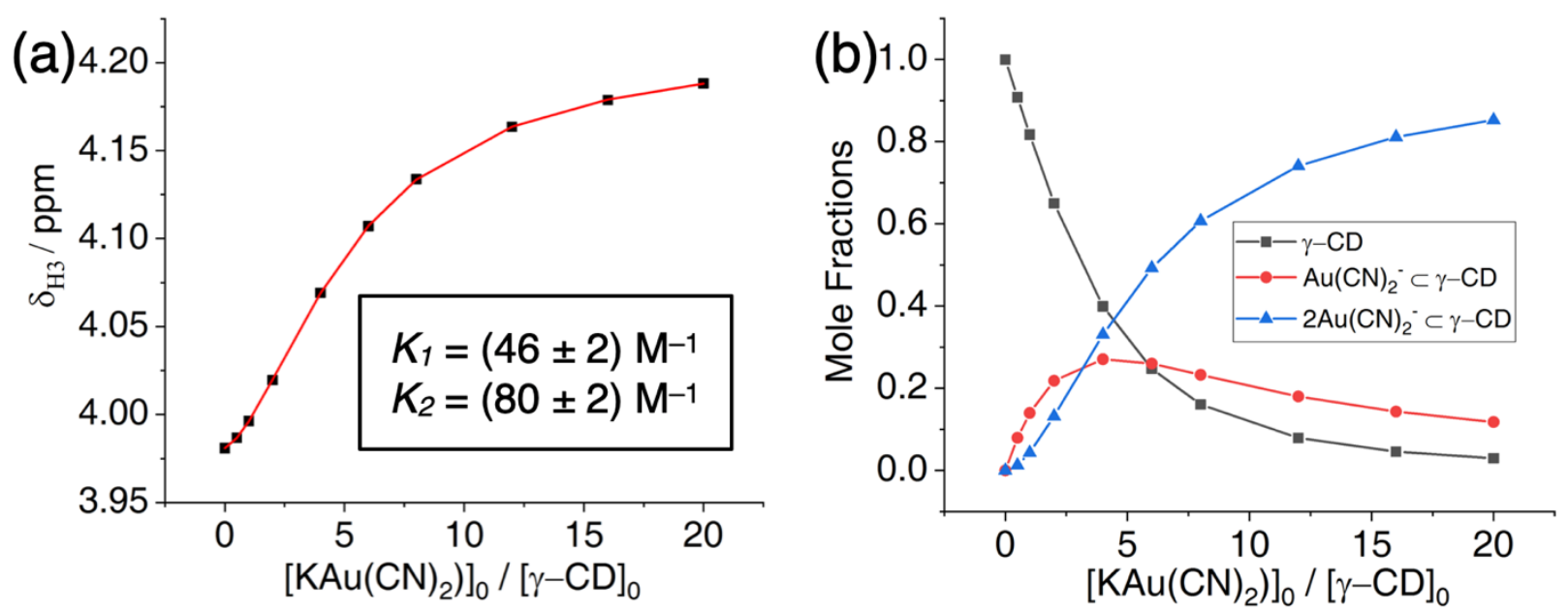

Figure S25. (a) Titration isotherm created by monitoring changes in the chemical shift of $\mathrm{H}-3$ for $\gamma-\mathrm{CD}(5 \mathrm{mM})$ caused by the addition of $\mathrm{KAu}(\mathrm{CN})_{2}$ at $25^{\circ} \mathrm{C}$. Red lines are curve fitting using a 1:2 receptor-substrate binding model. (b) Calculated changes of mole fractions for $\gamma-\mathrm{CD}, \mathrm{Au}(\mathrm{CN})_{2}{ }^{-} \subset$ $\gamma-\mathrm{CD}$ and $2 \mathrm{Au}(\mathrm{CN})_{2}{ }^{-} \subset \gamma-\mathrm{CD}$ over the substrate receptor mole ratio

\section{Section 5. Binding Studies Using Isothermal Titration Calorimetry}

Isothermal titration calorimetry (ITC) was performed by TA Nano Isothermal Titration Calorimeter at $25^{\circ} \mathrm{C}$. A Hastelloy cell was used with an active cell volume of $190 \mu \mathrm{L}$. The stirring speed was set at $75 \mathrm{rpm}$. Receptor and substrate solutions were prepared in Milli-Q water and allowed to equilibrate overnight if necessary. In each titration experiment, 20-25 injections were performed with gradually decreased titration peaks until saturation is reached, at which point only heat of dilution was measured. The heat of dilution, when using $\gamma-\mathrm{CD}$, was directly measured by titrating $\mathrm{KAu}(\mathrm{CN})_{2}$ into a blank solution. After subtracting the heat of dilution, the resulting data were analyzed with NanoAnalyze software using either a 1:1 or a 1:2 receptor-substrate binding model and plotted by Origin Lab 8.6 software. All the titrations were independently duplicated. Shown below is one set of titration isotherms. All isotherm fittings were used to calculate the average $K_{\mathrm{a}}$ and $\Delta H$ with relevant standard errors. 

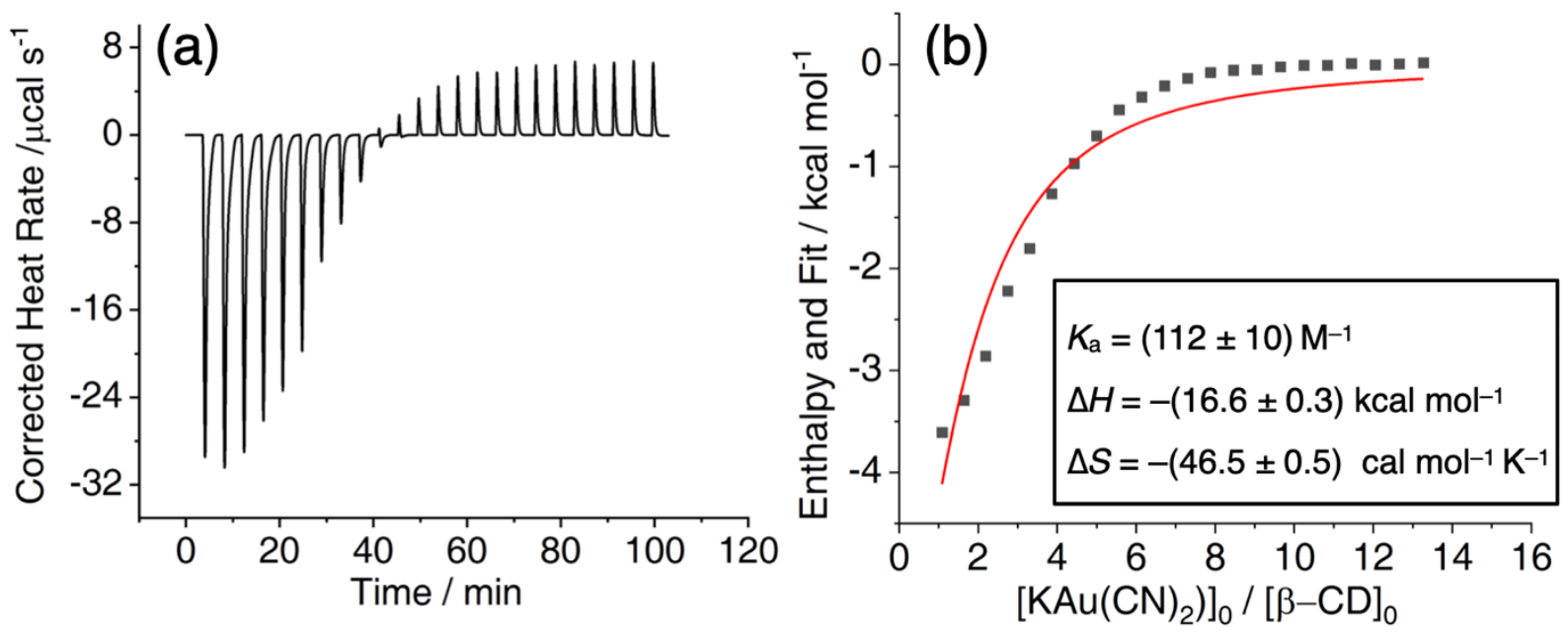

Figure S26. (a) ITC profile between $\mathrm{KAu}(\mathrm{CN})_{2}(500 \mathrm{mM}$, in the syringe $)$ and $\beta-\mathrm{CD}(5 \mathrm{mM}$, in the cell) at $25^{\circ} \mathrm{C}$ in $\mathrm{H}_{2} \mathrm{O}$. (b) Nonlinear fitting of enthalpy using 1:1 receptor-substrate binding model
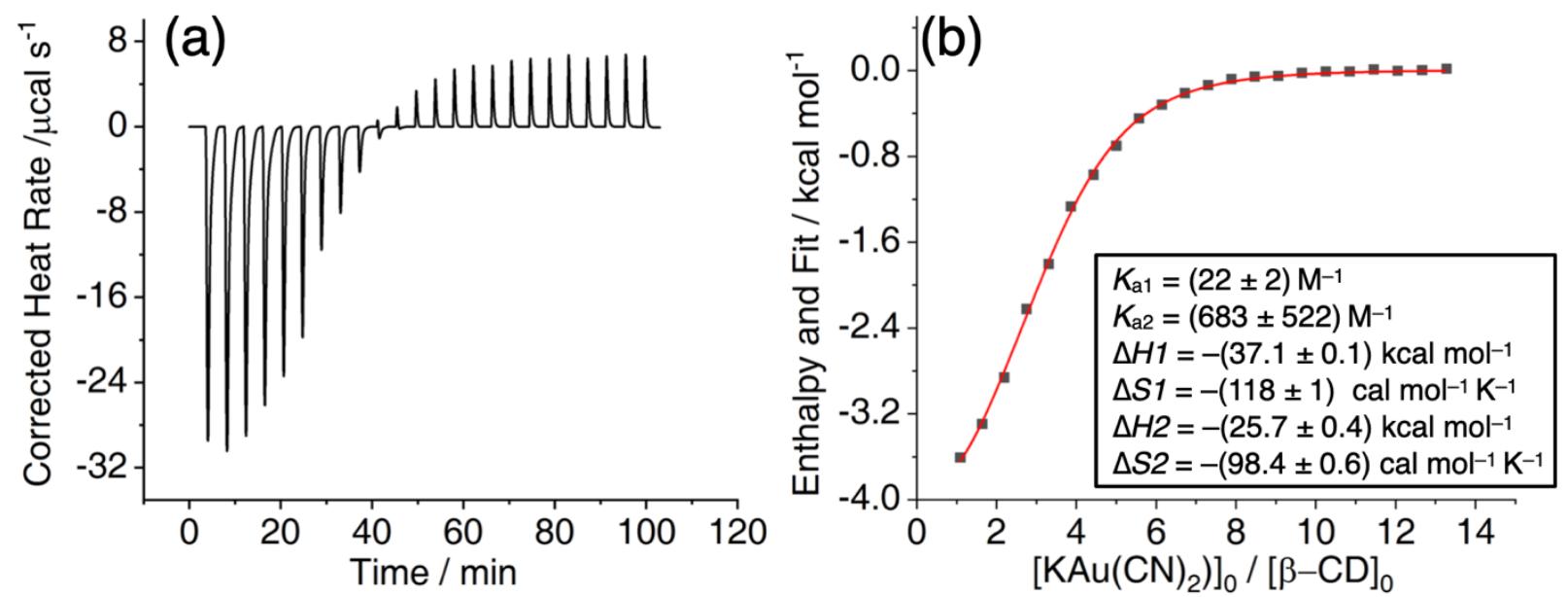

Figure S27. (a) ITC profile between $\mathrm{KAu}(\mathrm{CN})_{2}(500 \mathrm{mM}$, in the syringe $)$ and $\beta-\mathrm{CD}(5 \mathrm{mM}$, in the cell) at $25^{\circ} \mathrm{C}$ in $\mathrm{H}_{2} \mathrm{O}$. (b) Nonlinear fitting of enthalpy using a 1:2 receptor-substrate binding model 

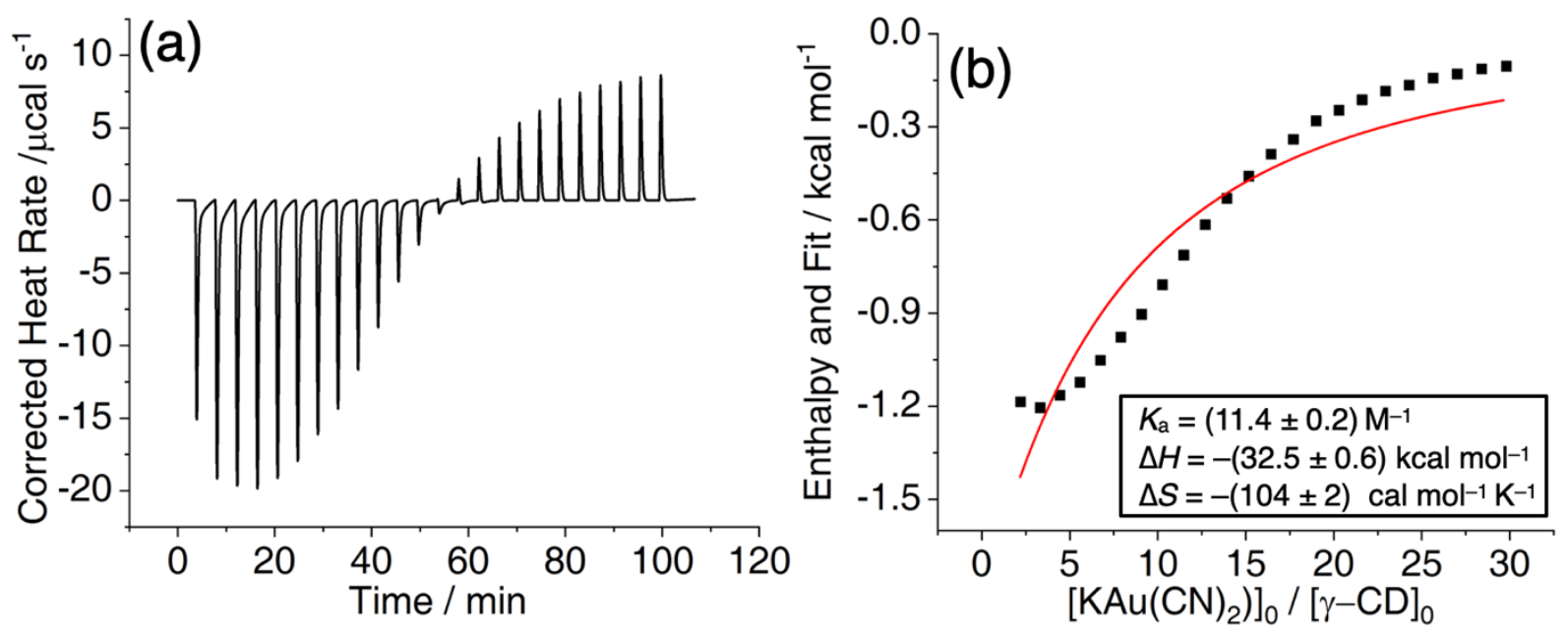

Figure S28. (a) ITC profile between $\mathrm{KAu}(\mathrm{CN})_{2}(500 \mathrm{mM}$, in the syringe) and $\gamma-\mathrm{CD}(5 \mathrm{mM}$, in the cell) at $25{ }^{\circ} \mathrm{C}$ in $\mathrm{H}_{2} \mathrm{O}$. (b) Nonlinear fitting of enthalpy using 1:1 receptor-substrate binding model
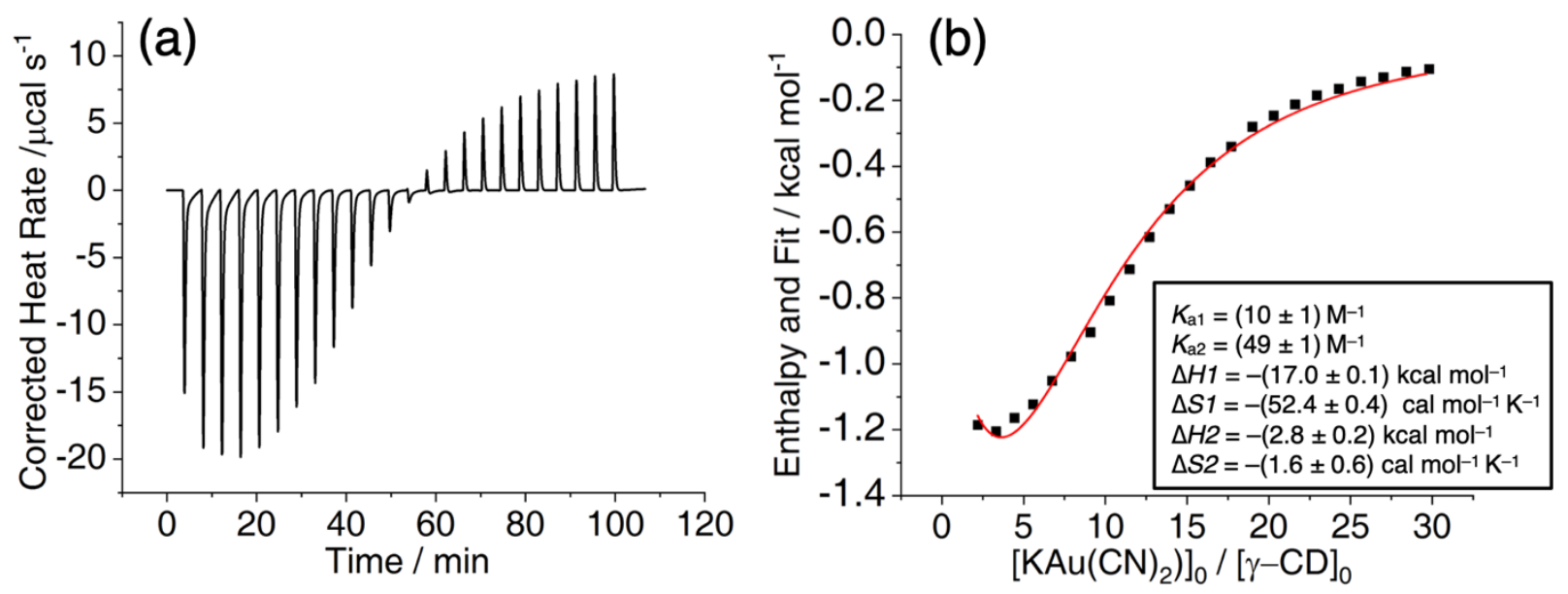

Figure S29. (a) ITC profile between $\mathrm{KAu}(\mathrm{CN})_{2}(500 \mathrm{mM}$, in the syringe) and $\gamma-\mathrm{CD}(5 \mathrm{mM}$, in the cell) at $25^{\circ} \mathrm{C}$ in $\mathrm{H}_{2} \mathrm{O}$. (b) Nonlinear fitting of enthalpy using a 1:2 receptor-substrate binding model 


\section{Section 6. Gold and Silver Stripping}

\section{Gold Adsorption on Activated Carbon}

Activated carbon $(10 \mathrm{~g})$ was added to an aqueous solution $(50 \mathrm{~mL})$ of $\mathrm{KAu}(\mathrm{CN})_{2}(150 \mathrm{mg}, \mathrm{Au}$ concentration: $2041 \mathrm{ppm}$ ), and the suspension was stirred at room temperature for 3 days. The activated carbon was filtrated and dried over the oven at $110{ }^{\circ} \mathrm{C}$ overnight. The remaining gold in the filtrate was analyzed by ICP, which revealed that the concentration of gold is less than 3 ppm, suggesting quantitative adsorption of Au on activated carbon. The activated carbon had a weight loss of 15\% after drying under the same conditions, indicating $102 \mathrm{mg}$ Au was adsorbed on $8.5 \mathrm{~g}$ activated carbon. The gold loading on activated carbon was thus calculated to be $12 \mathrm{mg} / \mathrm{g}$.

\section{Silver Adsorption on Activated Carbon}

Activated carbon $(10 \mathrm{~g})$ was added to an aqueous solution $(50 \mathrm{~mL})$ of $\mathrm{KAg}(\mathrm{CN})_{2}(150 \mathrm{mg}, \mathrm{Ag}$ concentration: $1613 \mathrm{ppm}$ ), and the suspension was stirred at room temperature for 3 days. The activated carbon was filtrated and dried in an oven at $110{ }^{\circ} \mathrm{C}$ overnight. The remaining gold in the filtrate was analyzed by ICP, which revealed that the concentration of gold is less than $3 \mathrm{ppm}$, suggesting quantitative adsorption of Ag on activated carbon. The activated carbon had a weight loss of 15\% after drying under the same conditions, indicating $88 \mathrm{mg} \mathrm{Ag}$ was adsorbed on $8.5 \mathrm{~g}$ activated carbon. The silver loading on activated carbon was estimated to be $10 \mathrm{mg} / \mathrm{g}$.

\section{Gold and Silver Adsorption on Activated Carbon}

Activated carbon $(10 \mathrm{~g})$ was added to an aqueous solution $(50 \mathrm{~mL})$ of $\mathrm{KAu}(\mathrm{CN})_{2}(150 \mathrm{mg}, \mathrm{Au}$ concentration: $2041 \mathrm{ppm}$ ) and $\mathrm{KAg}(\mathrm{CN})_{2}$ (150 mg, $\mathrm{Ag}$ concentration: $1613 \mathrm{ppm}$ ), and the suspension was stirred at room temperature for three days. The activated carbon was filtrated and dried in an oven at $110{ }^{\circ} \mathrm{C}$ overnight. The remaining gold and silver in the filtrate were analyzed by ICP, which revealed that the concentrations of gold and silver are less than 3 ppm, suggesting quantitative adsorption of $\mathrm{Au}$ and $\mathrm{Ag}$ on activated carbon. The activated carbon had a weight loss of $15 \%$ after drying under the same conditions, indicating $102 \mathrm{mg}$ Au and $88 \mathrm{mg} \mathrm{Ag}$ were adsorbed on $8.5 \mathrm{~g}$ activated carbon. The gold and silver loading on activated carbon was estimated to be 12 and $10 \mathrm{mg} / \mathrm{g}$, respectively. 


\section{Gold and /or Silver Stripping}

Metal loaded activated carbon ( $50 \mathrm{mg}$, containing either $0.6 \mathrm{mg} \mathrm{Au}$ or $0.5 \mathrm{mg} \mathrm{Ag}$ ) was added to an aqueous solution $(5 \mathrm{~mL})$ of $\alpha-\mathrm{CD}(0-10 \mathrm{w} / \mathrm{v} \%$, heat is applied to dissolve $\alpha-\mathrm{CD}$ when its concentration is $>2 \% \mathrm{w} / \mathrm{v} \%$ ). The suspension was stirred for $30 \mathrm{~min}$ at room temperature, after which the activated carbon was separated by centrifuge. The supernatant was filtrated by a 0.45 $\mu \mathrm{m}$ syringe filter, and the concentration of $\mathrm{Au}$ and $\mathrm{Ag}$ was analyzed by inductively coupled plasma mass spectrometry (ICP-MS). Each experiment was duplicated independently, and the average concentrations were presented with standard deviations. The same protocols were performed for gold stripping using $\gamma$-CD and selective gold string from the activated carbon loaded with both gold and silver.

\section{ICP-MS Analysis}

Quantification of gold $(\mathrm{Au})$ and silver (Ag) was accomplished using ICP-MS of acidified samples. Specifically, samples $(100 \mu \mathrm{L})$ designated for $\mathrm{Au}$ analysis were digested in concentrated $\mathrm{HNO}_{3}$ (200 uL, 69\%, Thermo Fisher Scientific, Waltham, MA, USA) and concentrated HCl (200 uL, 34\%, Thermo Fisher Scientific, Waltham, MA, USA) at room temperature for $10 \mathrm{~min}$ in a ventilated hood. Cyanide test strips were used to ensure that no cyanide gas was evolving after this time, and ultra-pure $\mathrm{H}_{2} \mathrm{O}(18.2 \mathrm{M} \Omega \cdot \mathrm{cm})$ was added to produce a final solution of $2.0 \% \mathrm{HNO}_{3}$ and $2.0 \% \mathrm{HCl}(\mathrm{v} / \mathrm{v})$ in a total sample volume of $10 \mathrm{~mL}$. A quantitative standard was made using a 100 $\mu \mathrm{g} / \mathrm{mL}$ Au elemental standard (Inorganic Ventures, Christiansburg, VA, USA), which was used to create a $100 \mathrm{ng} / \mathrm{g}$ Au standard in $2.0 \% \mathrm{HNO}_{3}$ and $2.0 \% \mathrm{HCl}(\mathrm{v} / \mathrm{v})$ in a total sample volume of 50 $\mathrm{mL}$. A solution of $2.0 \% \mathrm{HNO}_{3}$ and $2.0 \% \mathrm{HCl}(\mathrm{v} / \mathrm{v})$ was used as the calibration blank.

Samples designated for Ag analysis were digested in $200 \mathrm{uL}$ concentrated trace nitric acid $(>69 \%$, Thermo Fisher Scientific, Waltham, MA, USA) at room temperature for $10 \mathrm{~min}$ in a ventilated hood. Cyanide test strips were used to ensure that no cyanide gas was evolving after this time, and ultra-pure $\mathrm{H}_{2} \mathrm{O}(18.2 \mathrm{M} \Omega \cdot \mathrm{cm})$ was added to produce a final solution of $2.0 \% \mathrm{HNO}_{3}$ in a total sample volume of $10 \mathrm{~mL}$. A quantitative standard was made using a $100 \mu \mathrm{g} / \mathrm{mL} \mathrm{Ag} \mathrm{elemental}$ standard (Inorganic Ventures, Christiansburg, VA, USA), which was used to create a $100 \mathrm{ng} / \mathrm{g} \mathrm{Ag}$ standard in $2.0 \%$ nitric acid in a total sample volume of $50 \mathrm{~mL}$. A solution of $2.0 \%$ nitric acid was used as the calibration blank. 
ICP-MS was performed on a computer-controlled (QTEGRA software) Thermo iCapQ ICP-MS (Thermo Fisher Scientific, Waltham, MA, USA) operating in STD mode and equipped with an ESI SC-2DX PrepFAST autosampler (Omaha, NE, USA). The internal standard was added inline using the prepFAST system and consisted of $1 \mathrm{ng} / \mathrm{mL}$ of a mixed element solution containing Bi, In, ${ }^{6} \mathrm{Li}, \mathrm{Sc}, \mathrm{Tb}, \mathrm{Y}$ (IV-ICPMS-71D from Inorganic Ventures). Online dilution was also carried out by the prepFAST system and used to generate a calibration curve consisting of 100, 50, 10, 2 and $1 \mathrm{ppb} \mathrm{Au}$ or Ag. Each sample was acquired using 1 survey run (10 sweeps) and 3 main (peak jumping) runs (40 sweeps). The isotopes selected for analysis were ${ }^{107,109} \mathrm{Ag}$ or ${ }^{197} \mathrm{Au}$ and ${ }^{89} \mathrm{Y}$, ${ }^{115} \mathrm{In},{ }^{159} \mathrm{~Tb},{ }^{209} \mathrm{Bi}$ (chosen as internal standards for data interpolation and machine stability). Instrument performance is optimized daily through autotuning, followed by verification via a performance report (passing manufacturer specifications). 


\section{Section 7. References}

(S1) Dolomanov, O. V; Bourhis, L. J.; Gildea, R. J.; Howard, J. A. K.; Puschmann, H. OLEX2: A Complete Structure Solution, Refinement and Analysis Program. J. Appl. Crystallogr. 2009, 42, 339-341.

(S2) Sheldrick, G. M. SHELXT-Integrated Space-Group and Crystal-Structure Determination. Acta Crystallogr. Sect. A 2015, 71, 3-8.

(S3) Sheldrick, G. M. A Short History of SHELX. Acta Crystallogr. Sect. A 2008, 64, 112 122.

(S4) Thorn, A.; Dittrich, B.; Sheldrick, G. M. Enhanced Rigid-Bond Restraints. Acta Crystallogr. Sect. A 2012, 68, 448-451.

(S5) Lefebvre, C.; Rubez, G.; Khartabil, H.; Boisson, J. C.; Contreras-García, J.; Hénon, E. Accurately Extracting The Signature of Intermolecular Interactions Present in The NCI Plot of The Reduced Density Gradient Versus Electron Density. Phys. Chem. Chem. Phys. 2017, 19, 17928-17936.

(S6) Lu, T.; Chen, F. Multiwfn: A Multifunctional Wavefunction Analyzer. J. Comput. Chem. 2012, 33, 580-592.

(S7) Pettersen, E. F.; Goddard, T. D.; Huang, C. C.; Couch, G. S.; Greenblatt, D. M.; Meng, E. C.; Ferrin, T. E. UCSF Chimera - A Visualization System for Exploratory Research and Analysis. J. Comput. Chem. 2004, 25, 1605-1612.

(S8) Neese, F. The ORCA Program System. WIREs Comput. Mol. Sci. 2012, 2, 73-78.

(S9) Becke, A. D. Density-functional Thermochemistry. III. The Role of Exact Exchange. $J$. Chem. Phys. 1993, 98, 5648-5652.

(S10) Weigend, F.; Ahlrichs, R. Balanced Basis Sets of Split Valence \{,\} Triple Zeta Valence and Quadruple Zeta Valence Quality for H to Rn: Design and Assessment of Accuracy. Phys. Chem. Chem. Phys. 2005, 7, 3297-3305.

(S11) Neese, F. An Improvement of the Resolution of the Identity Approximation for the Formation of the Coulomb Matrix. J. Comput. Chem. 2003, 24, 1740-1747.

(S12) Izsák, R.; Neese, F. An Overlap Fitted Chain of Spheres Exchange Method. J. Chem. Phys. 2011, 135, 144105.

(S13) Stoychev, G. L.; Auer, A. A.; Neese, F. Automatic Generation of Auxiliary Basis Sets. J. Chem. Theory Comput. 2017, 13, 554-562.

(S14) Barone, V.; Cossi, M. Quantum Calculation of Molecular Energies and Energy Gradients in Solution by a Conductor Solvent Model. J. Phys. Chem. A 1998, 102, 1995-2001.

(S15) Thordarson, P. Determining Association Constants from Titration Experiments in Supramolecular Chemistry. Chem. Soc. Rev. 2011, 40, 1305-1323. 